ARTICLE

https://doi.org/10.1038/s41467-018-07835-1

\title{
Defect engineered bioactive transition metals dichalcogenides quantum dots
}

\author{
Xianguang Ding ${ }^{1,2}$, Fei Peng ${ }^{1}$, Jun Zhou ${ }^{3}$, Wenbin Gong ${ }^{4}$, Garaj Slaven (1) 2,3, Kian Ping Loh (1) 2,5, \\ Chwee Teck Lim (1D) $2,6,7,8$ \& David Tai Leong (1) ${ }^{1}$
}

Transition metal dichalcogenide (TMD) quantum dots (QDs) are fundamentally interesting because of the stronger quantum size effect with decreased lateral dimensions relative to their larger 2D nanosheet counterparts. However, the preparation of a wide range of TMD QDs is still a continual challenge. Here we demonstrate a bottom-up strategy utilizing TM oxides or chlorides and chalcogen precursors to synthesize a small library of TMD QDs $\left(\mathrm{MoS}_{2}, \mathrm{WS}_{2}, \mathrm{RuS}_{2}, \mathrm{MoTe}_{2}, \mathrm{MoSe}_{2}, \mathrm{WSe}_{2}\right.$ and $\left.\mathrm{RuSe}_{2}\right)$. The reaction reaches equilibrium almost instantaneously ( $10-20 \mathrm{~s}$ ) with mild aqueous and room temperature conditions. Tunable defect engineering can be achieved within the same reactions by deviating the precursors' reaction stoichiometries from their fixed molecular stoichiometries. Using $\mathrm{MoS}_{2}$ QDs for proof-of-concept biomedical applications, we show that increasing sulfur defects enhanced oxidative stress generation, through the photodynamic effect, in cancer cells. This facile strategy will motivate future design of TMDs nanomaterials utilizing defect engineering for biomedical applications.

\footnotetext{
${ }^{1}$ Department of Chemical and Biomolecular Engineering, National University of Singapore, Singapore 117585, Singapore. ${ }^{2}$ Centre for Advanced 2D Materials, Graphene Research Centre, National University of Singapore, Singapore 117546, Singapore. ${ }^{3}$ Department of Physics, National University of Singapore, Singapore 117542, Singapore. ${ }^{4}$ Division of Advanced Nanomaterials, Suzhou Institute of Nano-Tech and Nano-Bionics, Chinese Academy of Sciences, Suzhou 215123, China. ${ }^{5}$ Department of Chemistry, National University of Singapore, Singapore 117543 , Singapore. ${ }^{6}$ Department of Biomedical Engineering, National University of Singapore, Singapore 117575, Singapore. ${ }^{7}$ Mechanobiology Institute, Mechanobiology Institute, Singapore 117411, Singapore.

${ }^{8}$ Biomedical Institute for Global Health Research and Technology, Singapore 117599, Singapore. Correspondence and requests for materials should be addressed to C.T.L. (email: ctlim@nus.edu.sg) or to D.T.L. (email: cheltwd@nus.edu.sg)
} 
$\mathrm{T}$ wo-dimensional (2D) transition metal dichalcogenides (TMDs) with comparable structures to graphene, have attracted tremendous attention during the past few years. This large family of layered materials shared a common molecular formula and general structure where each member consists of mono-atomic thick stacked layers of repetitive covalently bonded $\mathrm{X}-\mathrm{M}-\mathrm{X}(\mathrm{M}=$ Transition metal; $\mathrm{X}=$ Chalcogen $)$. With decreasing number of layers, TMDs nanosheets transit from an indirect gap to a direct band-gap semi-conductor (e.g., $1.2-1.9 \mathrm{eV}$ for $\mathrm{MoS}_{2}$ from bulk form to monolayer) $)^{1,2}$. This special layersdependent physical property inspired 2D TMDs nanosheetsenabled applications in biomedicine ${ }^{3,4}$, sensors ${ }^{5}$, transistors $^{6}$, catalysts $^{7,8}$, photodetectors 9 , and energy storage devices ${ }^{10,11}$. Further reduction of the lateral size of TMDs few-layers or monolayer nanosheets to quantum dots (QDs) or 0D nanodots further accentuate their electrical/optical properties due to a stronger quantum confinement and edge effects ${ }^{12}$. This enhancement introduced more dimensions of interesting and exploitable properties for catalytic and biomedical applications ${ }^{13,14}$, of which the latter is gravely under-represented. This could be due to the harsh and non-biocompatible methods in robust synthesis of many of the TMD QDs.

Atomic defects in transition metal dichalcogenides (TMDs) nanomaterials add an additional dimension to its optical, chemical and electronic activities. Top-down approaches like laser, plasma and electron bombardment have been intensively used to introduce defects on TMDs nanosheets and thereby controlling their optical and electrical properties. Moreover, the methods are usually single sheet process, albeit with great precision but at the expense of low scalability and high cost. Applying the same top-down approaches to reliably engineer defects in TMD QDs is extremely difficult due to the minute size of the QDs. This lack of defect engineering option has further curtailed TMD QDs' potential.

In the past few years, many strategies, including lithium intercalation and exfoliation have been developed for synthesizing 2D TMD nanosheets ${ }^{15-17}$, but mild and general synthesis methods for 0D TMDs QDs are few. Top-down synthesis methods through a combination of grinding and sonication techniques, could obtain 1T-phase TMD QDs ${ }^{11,12}$. Although much progress has been achieved, to date, many more problems remain that needed solving before exploring their unique properties for catalytic and biomedical-based application. The topdown production of high quality TMD QDs has so far been extremely challenging because of the high degree of size and layers reduction required resulting in a low yield of production at the end of a long tedious process ${ }^{18,19}$, which involve timeconsuming exfoliation and ultrasonication processes starting from bulk TMD crystals, exfoliated first to 2D TMD nanosheets and then further cutting them down to TMD QDs sizes. Fastidious post-treatment processes (for example, gradient centrifugation) is required; further reducing the yields of each sized TMDs QDs. Reports on bottom-up strategies for TMD QDs are generally limited to harsh hydrothermal methods $\left(200^{\circ} \mathrm{C}\right.$ and at least $24 \mathrm{~h})^{20-22}$. Further defect engineering of as-synthesized TMD QDs will therefore allow for more property explorations of TMD QDs for catalytic, semi-conducting and biomedical applications.

Here we demonstrate a facile and possibly universal bottom-up route to synthesize $\mathrm{MoS}_{2}$ QDs with different degrees of defect under a fair degree of control. All the $\mathrm{MoS}_{2}$ QDs synthesized under our mild aqueous condition are of uniform sizes of around $3.9 \mathrm{~nm}$. This synthesis method has been further expanded to successfully prepare a library of various other TMD QDs. We also showed, using $\mathrm{MoS}_{2}$ QDs, its potential as a photodynamic agent that is easily tunable through bottom-up disordering engineering.
Previous studies have alluded to the possibility of defects in semiconductor QDs matter to photodynamic effect but that was never proven ${ }^{23}$, possibly due to the difficulty in controllable engineering defects in semiconductor QDs. In this paper, different degrees of defect engineering were achieved in 0D TMDs QDs via bottom-up stoichiometry deviations. With this defect-variable TMD QDs, the suspected correlation between defect degree and photodynamic properties is verified. The possible mechanism of defect enhanced reactive oxygen species (ROS) generation was proposed. By bottom-up defect engineering of TMD QDs, we showed that we could tune photodynamic oxidative stress effect on cancer cells.

\section{Results}

Characterization of as synthesized $\mathrm{MoS}_{2}$ QDs. The $\mathrm{MoS}_{2}$ QDs were prepared by a bottom-up route conducted in aqueous condition at room temperature via a simple chemical reaction starting with $\mathrm{Na}_{2} \mathrm{~S}$ and $\mathrm{MoCl}_{3}, \mathrm{MoCl}_{5}$ or $\mathrm{MoO}_{3}$. Inspired by biomineralization, natural biopolymers such as BSA were used as surfactant because of their template effect and excellent biocompatibility ${ }^{24,25}$. Briefly, $\mathrm{pH}$ value of Mo-precursor solution was firstly adjusted to be above 11. In this high $\mathrm{pH}$ solution, the molybdenum precursors $-\mathrm{MoCl}_{5}$ or $\mathrm{MoO}_{3}$ decompose, yielding stable $\mathrm{MoO}_{4}^{2-}$-(Supplementary Figure 1). The reaction scheme can be expressed as:

$$
\begin{gathered}
4 \mathrm{MoCl}_{5}+\mathrm{O}_{2}+6 \mathrm{H}_{2} \mathrm{O}+8 \mathrm{OH}^{-} \rightarrow 4 \mathrm{MoO}_{4}^{2-}+20 \mathrm{HCl} \\
\mathrm{MoO}_{3}+2 \mathrm{OH}^{-} \rightarrow \mathrm{MoO}_{4}^{2-}+\mathrm{H}_{2} \mathrm{O}
\end{gathered}
$$

Then they were transferred into BSA solution; $\mathrm{Na}_{2} \mathrm{~S}$ solution was introduced into the mixture. Subsequent adjusting of $\mathrm{pH}$ value to 6 with addition of $\mathrm{HCl}$ activates the sulfur precursors, initiating the reaction between $\mathrm{MoO}_{4}{ }^{2-}$ and sulfur precursors protected by BSA (Fig. 1a, Supplementary Movie 1). Our one-pot reaction was carried out under mild experimental conditions in the laboratory; without the need for high temperature and pressure or special apparatus ${ }^{26}$. Our method is easily scalable. The aqueous product showed a faint yellow color with good dispersibility (Fig. 1b). The transmission electron microscopy (TEM) images showed a consistent size quality of the as synthesized $\mathrm{MoS}_{2}$ QDs to be $\sim 3.9 \mathrm{~nm}$ (Fig. 1c). The observed d-spacing $(0.27 \mathrm{~nm})$ is assigned to the (100) atomic plane of $\mathrm{MoS}_{2}$, confirming the high crystallinity of the $\mathrm{MoS}_{2}$ QDs (Fig. 1d). X-ray photoelectron spectroscopy (XPS) was also performed to measure the composition and the corresponding chemical valence of the elements in $\mathrm{MoS}_{2}$ QDs (Supplementary Figure 2). Typical $\mathrm{Mo}^{4+} 3 \mathrm{~d}_{3 / 2}$ peaks of $232.8 \mathrm{eV}$ and $\mathrm{Mo}^{4+} 3 \mathrm{~d}_{5 / 2}$ peaks of $229.6 \mathrm{eV}$ could be clearly observed from the XPS spectrum (Supplementary Figure 2a), which suggested the presence of Mo (IV) of $\mathrm{MoS}_{2} \mathrm{QDs}^{27}$. Supplementary Figure $2 \mathrm{~b}$ shows the binding energy of sulfur with the $\mathrm{S} 2 \mathrm{p}_{3 / 2}$ and $\mathrm{S}$ $2 \mathrm{p}_{1 / 2}$ peaks at $162.15 \mathrm{eV}$ and $163.05 \mathrm{eV}$, which is consistent with -2 oxidation state of sulfur. The atomic ratio of Mo to sulfur was quantified to be 1: 2.31 . The higher sulfur content compared to the initial feed ratio (Mo: $S=1: 2$ ) might be due to the presence of S- containing cysteines in BSA on the surface of $\mathrm{MoS}_{2}$ QDs. The structure of $\mathrm{MoS}_{2}$ QDs was further characterized by powder X-Ray diffractometry (XRD, Fig. 1e). The XRD pattern of $\mathrm{MoS}_{2}$ QDs shows broad diffraction peaks, which is in accordance with the XRD features of low-dimensional nanoparticles, suggesting the small grain sizes of the $\mathrm{MoS}_{2}$ QDs. The main peaks at $11^{\circ}, 29^{\circ}$ and $36^{\circ}$ can be assigned to the characteristic (001), (100) and (103) planes of hexagonal 2H-MoS (JCPDS NO. 24-0513). The colloidal suspension of $\mathrm{MoS}_{2}$ QDs prepared was very stable, with no obvious aggregations over at least 3 months (Supplementary Figure 3). This high stability property is an important advantage 
a

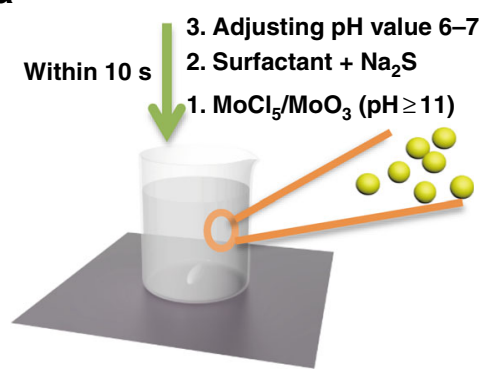

b

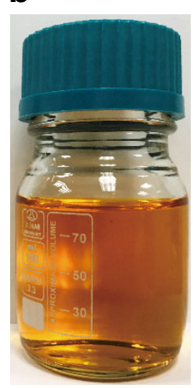

C

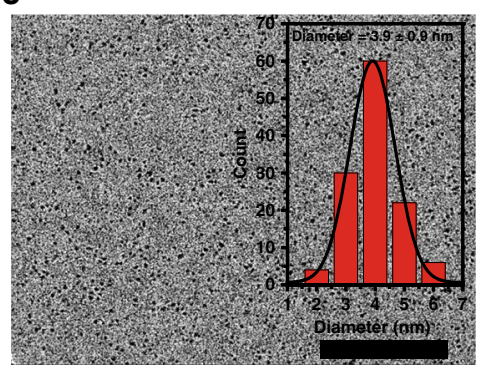

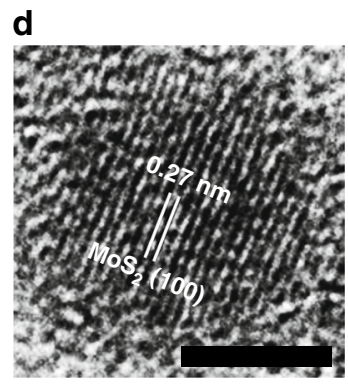

e

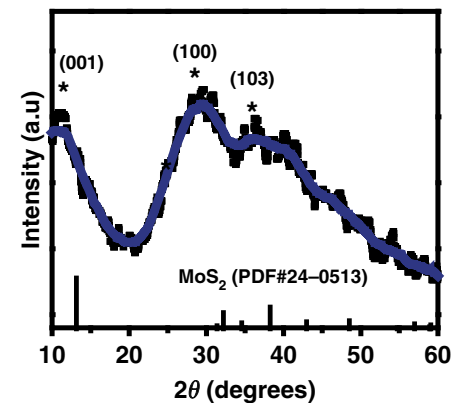

f

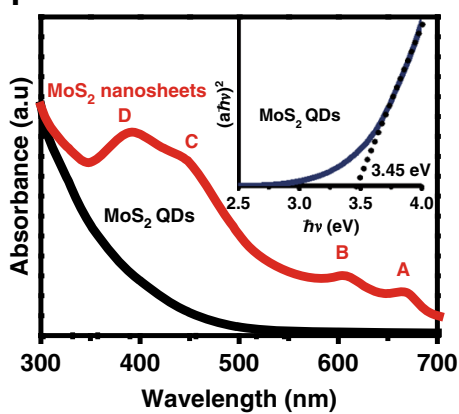

Fig. 1 Benign aqueous room temperature bottom-up synthesis of $\mathrm{MoS}_{2}$ QDs. a Preparation of $\mathrm{MoS}_{2}$ QDs with bottom-up strategy. b Easy and scalable aqueous $\mathrm{MoS}_{2}$ colloidal suspension. Synthesis step takes less than 10-20 s (also refer to Supplementary Movie 1). c Ultrasmall but consistent assynthesized $\mathrm{MoS}_{2}$ QDs. Inset: Distribution of $\mathrm{MoS}_{2}$ QDs size measured (ImageJ, $n=200$ ). Scale bar: $100 \mathrm{~nm}$. d HRTEM (Scale bar: $5 \mathrm{~nm}$ ), e XRD and f UV-vis absorption spectra of $M_{0} S_{2}$ QDs showing a distinctively different nanomaterial from exfoliated $M_{0} S_{2}$ nanosheets

in biomedical applications. In comparison to $2 \mathrm{D} \mathrm{MoS}_{2}$ nanosheets with relatively large lateral dimensions, four distinct excitonic peaks from $2 \mathrm{D} \mathrm{MoS}$ nanosheets $(\mathrm{A}, \mathrm{B}, \mathrm{C}$ and $\mathrm{D})$ were absent in the optical spectrum of $\mathrm{MoS}_{2}$ QDs (Fig. 1f), along with strongly absorption blue-shifted towards shorter wavelengths. These optical features should result from the stronger quantum size effect of $\mathrm{MoS}_{2}$ QDs with decreased lateral dimensions relative to $2 \mathrm{D} \mathrm{MoS}_{2}$ nanosheets (Supplementary Figure 4). Noticeably, $\mathrm{MoS}_{2}$ QDs exhibited a new absorption peak at around $310 \mathrm{~nm}$. Similar absorption features were also observed by others ${ }^{28,29}$; attributed to their quantum confinement effect. Here the as-prepared $\mathrm{MoS}_{2}$ QDs had an average diameter of $3.9 \mathrm{~nm}$, which is close to the bulk Bohr exciton diameter of $\sim 4 \mathrm{~nm}^{30}$. The discretized bands induced by quantum confinement allowed transitions from the deep valance band to the conduction band, increasing the discrete absorption bands of $\mathrm{MoS}_{2}$ QDs. By using the method based on the relation of $(\alpha \hbar v)^{2}$ versus $\hbar v$ ( $\alpha$ is absorbance, $\hbar$ is the Planck's constant and $v$ is frequency), the optical band gap of our $\mathrm{MoS}_{2}$ QDs was calculated to be $\sim 3.45 \mathrm{eV}$, which is higher than that of bulk and single-layer $\mathrm{MoS}_{2}(1.2-1.9 \mathrm{eV})$. The quantum confinement effect and surface states (like surface defects) induced by the bottom-up method in $\mathrm{MoS}_{2}$ QDs may have collectively contributed to this band gap. With the presence of localized defect states, the surface states can narrow the calculated optical band gap through generating band-tailing effects ${ }^{31-33}$.

Surfactant effect on quality of $\mathrm{MoS}_{2}$ QDs. The hydrodynamic diameter of $\mathrm{MoS}_{2}$ QDs was determined to be $13 \mathrm{~nm}$ by dynamic light scattering (DLS), which was slightly larger than that of BSA $(10 \mathrm{~nm})$ and significantly higher than the TEM size of $\sim 3.9 \mathrm{~nm}$ (Fig. 2a). One

$\mathrm{MoS}_{2}$ QD is likely to be interacting with one BSA molecule (Fig. 2b). The estimation value from thermogravimetric analysis was slightly larger than the DLS result (Supplementary Figure 5).
With BSA as template to confine the growth of $\mathrm{MoS}_{2}$ QDs, the biomineralization process allow good size quality control. To investigate the role that the surfactant plays in the quality of $\mathrm{MoS}_{2}$ QDs, we also tested gluconate (Glu), poly-arginine (Poly-Arg) and cysteine (Cys) in the direct synthesis method. These biomolecules were selected as they provide the representative functional groups of BSA respectively: in Glu $(-\mathrm{COOH})$, in PolyArg $\left(-\mathrm{NH}_{2}\right)$, in Cys $\left(-\mathrm{SH},-\mathrm{COOH},-\mathrm{NH}_{2}\right)$. Figure $2 \mathrm{c}$ suggests that all of above surfactants could mediate the successful synthesis of $\mathrm{MoS}_{2}$ QDs, while particle quality is highly dependent on the surfactant of choice. To quantify the particle size distribution, full width at half maximum (FWHM) was counted by analyzing the poly-distribution of $\mathrm{MoS}_{2}$ QDs from different surfactants. The size distribution and poly-distribution were analyzed from TEM samples with over 200 particles (with ImageJ). The statistical FWHM values of $\mathrm{MoS}_{2}$ QDs from BSA, Cys, Glu and Poly-Arg were $1.8,3.1,5.8$ and 7.2 , with the mean sizes of 3.9, 7.4, 7.3 and $9 \mathrm{~nm}$, respectively (Fig. 2d), suggestive that BSA was more favorable for smaller QDs size and size distribution control. This also ruled out the sole fine size control of $-\mathrm{COOH},-\mathrm{NH}_{2}$ and -SH groups alone. Previous research suggested that disulfide bonds might possess much stronger binding energy to $2 \mathrm{D} \mathrm{MoS}_{2}$ compared to single thiol ${ }^{34,35}$. To check that possibility in our $\mathrm{MoS}_{2}$ QDs, first-principle calculation was carried out to verify the binding affinity of the major functional groups of BSA to $\mathrm{MoS}_{2}$ QDs. As shown in Fig. 2e, benzene rings and disulfide exhibited much higher binding energy to $\mathrm{MoS}_{2}$ QDs compared to $-\mathrm{SH}$, $-\mathrm{COOH},-\mathrm{SH},-\mathrm{NH}_{2}$ and $-\mathrm{OH}$ groups, which is $1.3 \mathrm{eV}$ and $0.84 \mathrm{eV}$ with respect to that of other groups $(0.46 \mathrm{eV}$ for $-\mathrm{SH}$, $0.45 \mathrm{eV}$ for $-\mathrm{NH}_{2}$ and $0.51 \mathrm{eV}$ for $-\mathrm{COOH}$ ) (Supplementary Table 1). These simulation results were consistent with the statistic FWHM values from TEM images (Fig. 2c), indicating that benzene rings and disulfide bonds may provide stronger affinity to stabilize QDs against aggregation and confine them for homogeneous growth, thus encouraging overall seeding and 

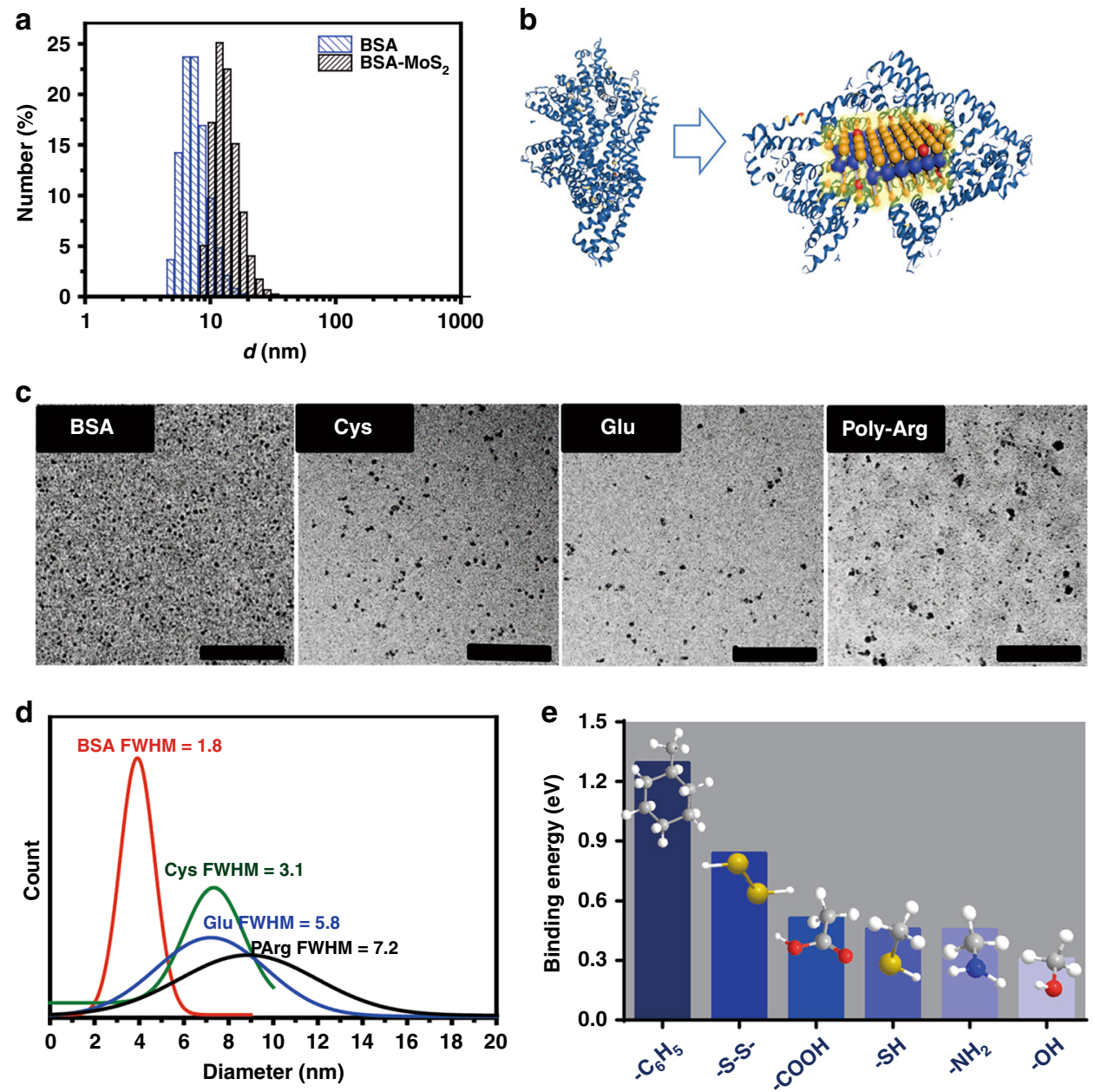

Fig. 2 Surfactant effect on quality of $M_{0} S_{2}$ QDs. a Size of the BSA and MoS 2 QDs determined by DLS. b Schematic illustration of possible BSA interactions with one $\mathrm{MoS}_{2}$ QD. c TEM images of MoS 2 QDs synthesized with BSA, Cys, Glu and Poly-Arg. Scale bar: $200 \mathrm{~nm}$. d Statistical analysis of the size distributions of $\mathrm{MoS}_{2}$ QDs synthesized with different biological surfactants. e Binding energy of major functional groups of BSA to MoS 2 QDs

growth process in our case. To get direct experimental evidence that disulfide bonds does make a significant difference in the synthesis of $\mathrm{MoS}_{2}$ QDs, disulfide bridges of BSA was first reduced with $\mathrm{NaBH}_{4}$ to sulfhydryl groups. These denatured BSA (dBSA) with thiol groups were then used to prepare $\mathrm{MoS}_{2}$ QDs as per our synthesis protocol. The size of the as-acquired $\mathrm{MoS}_{2}$ QDs using dBSA exhibited a significant increase from $3.9 \mathrm{~nm}$ to a mean size of $6.3 \mathrm{~nm}$ (Supplementary Figure 6). This result was similar to Cys mediated samples which are laden with thiol groups, verifying the significant effect of disulfide bonds on the fine size distribution control of $\mathrm{MoS}_{2}$ QDs. The effect of benzene rings was also tested by using 2, 5 dihydroxybenzoic acid (DBA) as a stabilizer to synthesize $\mathrm{MoS}_{2}$-DBA. However, compared with other surfactants, the morphology of $\mathrm{MoS}_{2}$-DBA QDs was less controllable with the FWHM value to be 13.4 (Supplementary Figure 7), possibly due to the strong van der Waals interactions between benzene rings and weak ions-benzene ring interactions in the seeding stage of the particle formation. From these results we can conclude that the optimal surfactant for synthesizing $\mathrm{MoS}_{2}$ QDs are molecules containing disulfide bonds such as BSA.

The formation of $\mathrm{MoS}_{2}$ QDs was very fast at relatively low energy, as observed by TEM images sampled at different reaction time points and synthesis temperature (Supplementary Figure 8 and 9). The acquired $\mathrm{MoS}_{2}$ QDs could be easily purified by the addition of $\mathrm{Cu}^{2+}$ solution to precipitate out the $\mathrm{MoS}_{2}$ QDs, followed by centrifugation and dialysis to remove the supernatant and ions ${ }^{36}$ (Supplementary Figure 10), thus avoiding the fussy and time-consuming post-treatment process.

Expansion of this synthetic strategy to other TMD QDs. We expanded this facile and mild bottom-up method to prepare a variety of TMD QDs based on the straightforward chemical

reaction between chalcogen precursor and transition metal salt such as metallic oxide or metallic chloride (Fig. 3a), which may be applied to synthesize various TMD QDs (Fig. 3b). To validate the feasibility of this method, BSA was applied as the surfactant for the proof-of-concept verification. Different transition metal sources (for example Mo, W and $\mathrm{Ru}$ ) and chalcogenide source ( $\mathrm{S}, \mathrm{Se}$ and $\mathrm{Te}$ ) were mixed with BSA at the $\mathrm{pH}$ value of 11 . As suggested in Fig. 4, after $\mathrm{pH}$ value was adjust to $6 \sim 7 . \mathrm{WS}_{2}, \mathrm{RuS}_{2}$, $\mathrm{MoTe}_{2}, \mathrm{MoSe}_{2}, \mathrm{WSe}_{2}$ and $\mathrm{RuSe}_{2}$ were successfully prepared. The TEM clearly illustrated their highly homogeneous of size distribution with majority of the diameters to be below $10 \mathrm{~nm}$. The corresponding images of different TMD QDs suspensions in $\mathrm{H}_{2} \mathrm{O}$ showed good dispersibility. Because of different bandgaps and sizes, the prepared TMD QDs library showed different colors in aqueous solution and exhibited correspondingly different UV-Vis

absorption spectra (Supplementary Figure 11). It is worth noting that some rod-like shape can also be found in the TEM 
a

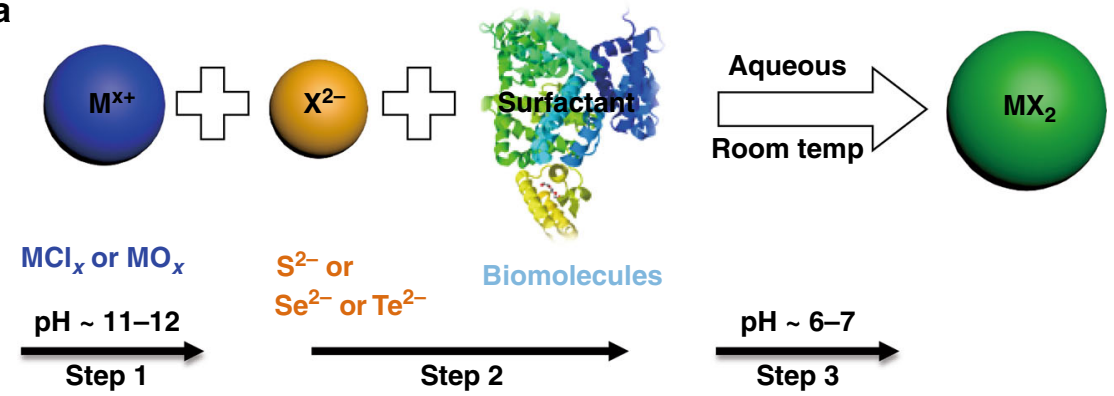

b

\begin{tabular}{|c|c|c|c|c|c|c|c|c|c|c|c|c|c|c|c|c|}
\hline \multirow{3}{*}{$\begin{array}{c}\mathrm{H} \\
\mathrm{Li} \\
\mathrm{Na}\end{array}$} & & \multirow{2}{*}{\multicolumn{9}{|c|}{$\begin{array}{l}M_{2} \\
M(\text { Transition metal })=\text { Mo, W, Ti... } \\
X(\text { Chalcogen })=S, S e, T e\end{array}$}} & \multirow{3}{*}{$\begin{array}{l}\mathrm{B} \\
\mathrm{Al}\end{array}$} & \multirow{3}{*}{$\frac{\mathrm{C}}{\mathrm{Si}}$} & \multirow{3}{*}{$\mathrm{N}$} & \multirow{3}{*}{$\begin{array}{l}0 \\
s\end{array}$} & \multirow{3}{*}{$\frac{\mathrm{F}}{\mathrm{Cl}}$} & \multirow{3}{*}{$\begin{array}{l}\mathrm{He} \\
\mathrm{Ne} \\
\mathrm{Ar}\end{array}$} \\
\hline & \multirow{2}{*}{\begin{tabular}{|l}
$\mathrm{Be}$ \\
$\mathrm{Mg}$
\end{tabular}} & & & & & & & & & & & & & & & \\
\hline & & 3 & 4 & 5 & 6 & & 9 & 10 & 11 & 12 & & & & & & \\
\hline $\mathrm{K}$ & $\mathrm{Ca}$ & Sc & $\mathrm{Ti}$ & v & $\mathrm{Cl}_{1}$ & & Co & $\mathrm{Ni}$ & $\mathrm{Cu}$ & $\mathrm{Zn}$ & $\mathrm{Ga}$ & $\mathrm{Ge}$ & As & $\mathrm{Se}$ & $\mathrm{Br}$ & $\mathrm{Kr}$ \\
\hline $\mathrm{Rb}$ & $\mathrm{Sr}$ & $Y$ & $\mathrm{Zr}$ & $\mathrm{Nb}$ & Mc & & Rh & Pd & $\mathrm{Ag}$ & $\mathrm{Cd}$ & In & Sn & $\mathrm{Sb}$ & $\mathrm{Te}$ & 1 & $\mathrm{Xe}$ \\
\hline Cs & $\mathrm{Ba}$ & La-Lu & Hf & Ta & w & & Ir & Pt & $\mathrm{Au}$ & $\mathrm{Hg}$ & $\mathrm{TI}$ & $\mathrm{Pb}$ & $\mathrm{Bi}$ & Po & At & $\mathrm{Rn}$ \\
\hline $\mathrm{Fr}$ & Ra & Ac-Lr & $\mathrm{Rf}$ & $\mathrm{Db}$ & $\mathrm{Sc}$ & & Mt & Ds & $\mathrm{Rg}$ & Cn & Uut & $\mathrm{FI}$ & Uup & LV & Uus & Uno \\
\hline
\end{tabular}

Fig. 3 Illustration of the bottom-up synthesis of TMD QDs under mild condition. a In this study, only the synthesis of commonly reported TMDs $\left(\mathrm{MoS}_{2}, \mathrm{WS}_{2}, \mathrm{RuS}_{2}, \mathrm{MoTe}_{2}, \mathrm{MoSe}_{2}, \mathrm{WSe}_{2}\right.$ and RuSe $\mathrm{R}_{2}$ QDs were showed. b Extrapolating the synthesis method to other TMD QDs synthesis may present an universal method to synthesis the various TMDs QDs

images of $\mathrm{MoTe}_{2}$ samples, possibly due to high reactivity and instability of $\mathrm{MoTe}_{2}$ compounds compared to $\mathrm{MoS}_{2} / \mathrm{MoSe}_{2}$ compounds ${ }^{37}$.

Stoichiometry and characterization. As our approach is bottomup, the relative molarity of the reagents can be varied to be different from their stoichiometric ratios, enabling defect creation or dopant incorporation ${ }^{38}$. This theoretically allowed us to engineer defects in the as-synthesized TMD QDs from the bottom-up manner, thus providing a window of opportunity to tune their optical and electrical properties at the synthesis stage. Using $\mathrm{MoS}_{2}$ QDs as an example, three $\mathrm{Mo} / \mathrm{S}$ relative molar concentrations, (4:2, 4:4 and 4:8) were pre-determined and reacted. Their respective products were named as $M_{0} S_{2}-D_{H}, M o S_{2}-D_{M}$ and $\mathrm{MoS}_{2}-\mathrm{D}_{\mathrm{L}}$ respectively. As shown in Fig. 5a, with increasing sulfur amount, the sample color changed from pale yellow for $\mathrm{MoS}_{2}-\mathrm{D}_{\mathrm{H}}$ to yellow and orange for $\mathrm{MoS}_{2}-\mathrm{D}_{\mathrm{M}}$ and $\mathrm{MoS}_{2}-\mathrm{D}_{\mathrm{L}}$, respectively. Careful size analysis from TEM images ( 200 particles counted) from the three groups indicated that varying initial relative concentration of reagents did not change the size distributions of the as-synthesized $\mathrm{MoS}_{2}$ QDs. Three $\mathrm{MoS}_{2}$ QDs groups exhibited similar size distributions with the average diameter of around $3.9 \mathrm{~nm}$ (Fig. 5b). The HRTEM images showed obvious discontinuities on the lattice planes such as dislocations and distortions were observed in the groups with the off-stoichiometric $\mathrm{MoS}_{2} \mathrm{~S}$ mole ratios like $\mathrm{MoS}_{2}-\mathrm{D}_{\mathrm{H}}$ and $\mathrm{MoS}_{2}-\mathrm{D}_{\mathrm{M}}$ QDs group (Fig. 5b). This discontinuity of the local lattice was thought to be caused by defects within crystals ${ }^{39}$. In $\mathrm{MoS}_{2}-\mathrm{D}_{\mathrm{H}}$ HRTEM images, several lattice planes with more discontinuities presented in the $\mathrm{MoS}_{2}$ structures, suggesting the strongly disordered

arrangement of nanodomains in $\mathrm{MoS}_{2}-\mathrm{D}_{\mathrm{H}}$ samples (Fig. 5b). When the sulfur amount increases approaching stoichiometric $\mathrm{MoS}_{2}$, relatively perfect single crystals without disorder were observed, suggesting the relatively fewer defects in $\mathrm{MoS}_{2}-\mathrm{D}_{\mathrm{L}}$. (Fig. 5b) The XRD diffraction peaks in three samples were broadening, confirming the nanoscale of these crystallites in different dimensions (Fig. 5c). A slight shift of major diffraction peaks indexed to (100) and (103) toward lower angles could also be observed from $\mathrm{MoS}_{2}-\mathrm{D}_{\mathrm{H}}$ to $\mathrm{MoS}_{2}-\mathrm{D}_{\mathrm{L}}$ samples, indicating the gradually enlarged lattice constants with increasing sulfur amounts and the homogeneous phase structures of all three $\mathrm{MoS}_{2}$ QDs groups. The stoichiometry related surface states were also investigated by studying the PL behavior of three $\mathrm{MoS}_{2}$ QDs. All three defect states exhibited excitation-dependent PL behaviors (Supplementary Figure 12). Their representative PL spectrum, with each emission spectrum were normalized to their solution optical absorbance density at the PL excitation wavelength of $400 \mathrm{~nm}$ (Fig. 5d). Such normalization was used to highlight the PL intensity change. By integrating the respective peak areas, we observed that the quantum yield (QY) of $\mathrm{MoS}_{2}-\mathrm{D}_{\mathrm{H}}$ (least sulfur content) possess the highest QY, which is approximately 1.79 times more than that of $\mathrm{MoS}_{2}-\mathrm{D}_{\mathrm{M}}$ (medium sulfur content) and 2.42 times higher than that of $\mathrm{MoS}_{2}-\mathrm{D}_{\mathrm{L}}$. Previous reports suggested that the surface state of nanomaterial is similar to a molecular state. Both the surface state (such as defects) and intrinsic state (quantum size effect) of nanomaterial contribute to the complexity of excited states of QDs ${ }^{40,41}$. The TEM analysis of the lattice planes disorder appears to point to $\mathrm{MoS}_{2}-\mathrm{D}_{\mathrm{H}}$ as having the greatest degree of defects (Fig. 5). Interestingly, we observed a trend that with more defects, the photoluminescence quantum yield also increased. Moreover, the $\mathrm{MoS}_{2}$ QDs show two emission peaks with one at around $463-478 \mathrm{~nm}$ and the other one located at $530 \mathrm{~nm}$, which could be attributed to intrinsic state emission (electron-hole recombination) and defect state emission, respectively ${ }^{29}$. In general, both intrinsic and defect state emissions influence the fluorescence spectrum. The red shift of fluorescence peaks from $463 \mathrm{~nm}$ of $\mathrm{MoS}_{2}-\mathrm{D}_{\mathrm{L}}$ to $469 \mathrm{~nm}$ for $\mathrm{MoS}_{2}-\mathrm{D}_{\mathrm{M}}$ and $478 \mathrm{~nm}$ for $\mathrm{MoS}_{2}-\mathrm{D}_{\mathrm{H}}$ can be observed and possibly due to the gradually increasing defects from $\mathrm{MoS}_{2}-\mathrm{D}_{\mathrm{L}}$ to $\mathrm{MoS}_{2}-\mathrm{D}_{\mathrm{H}}$. 

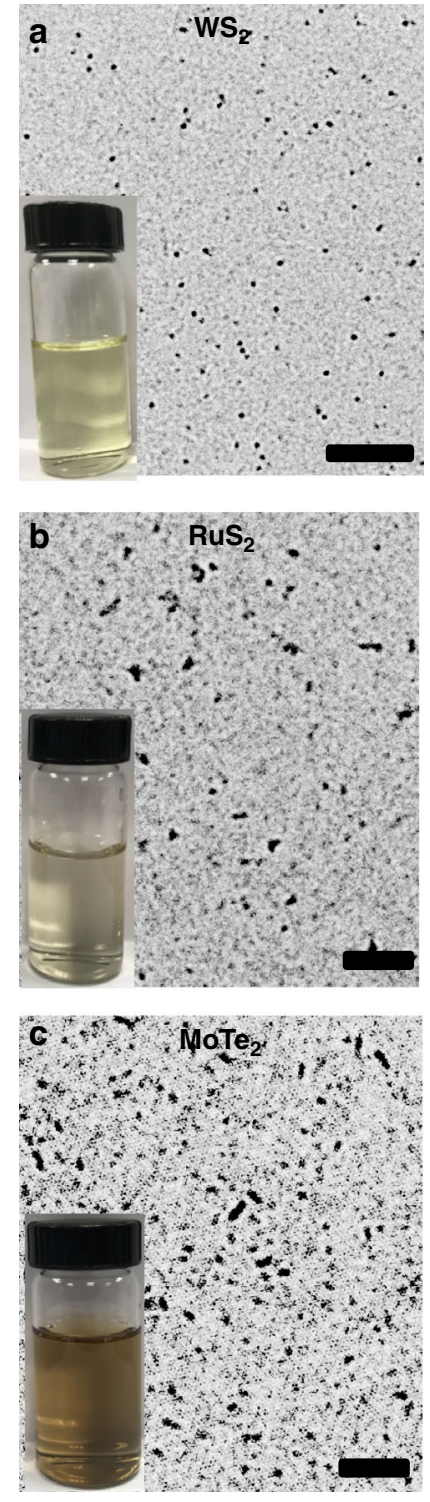
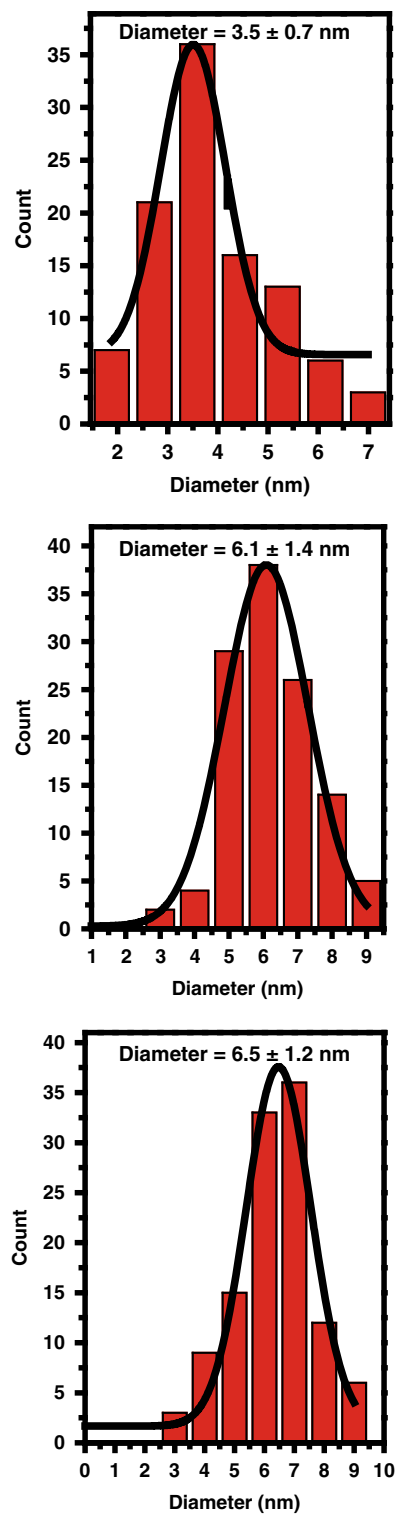
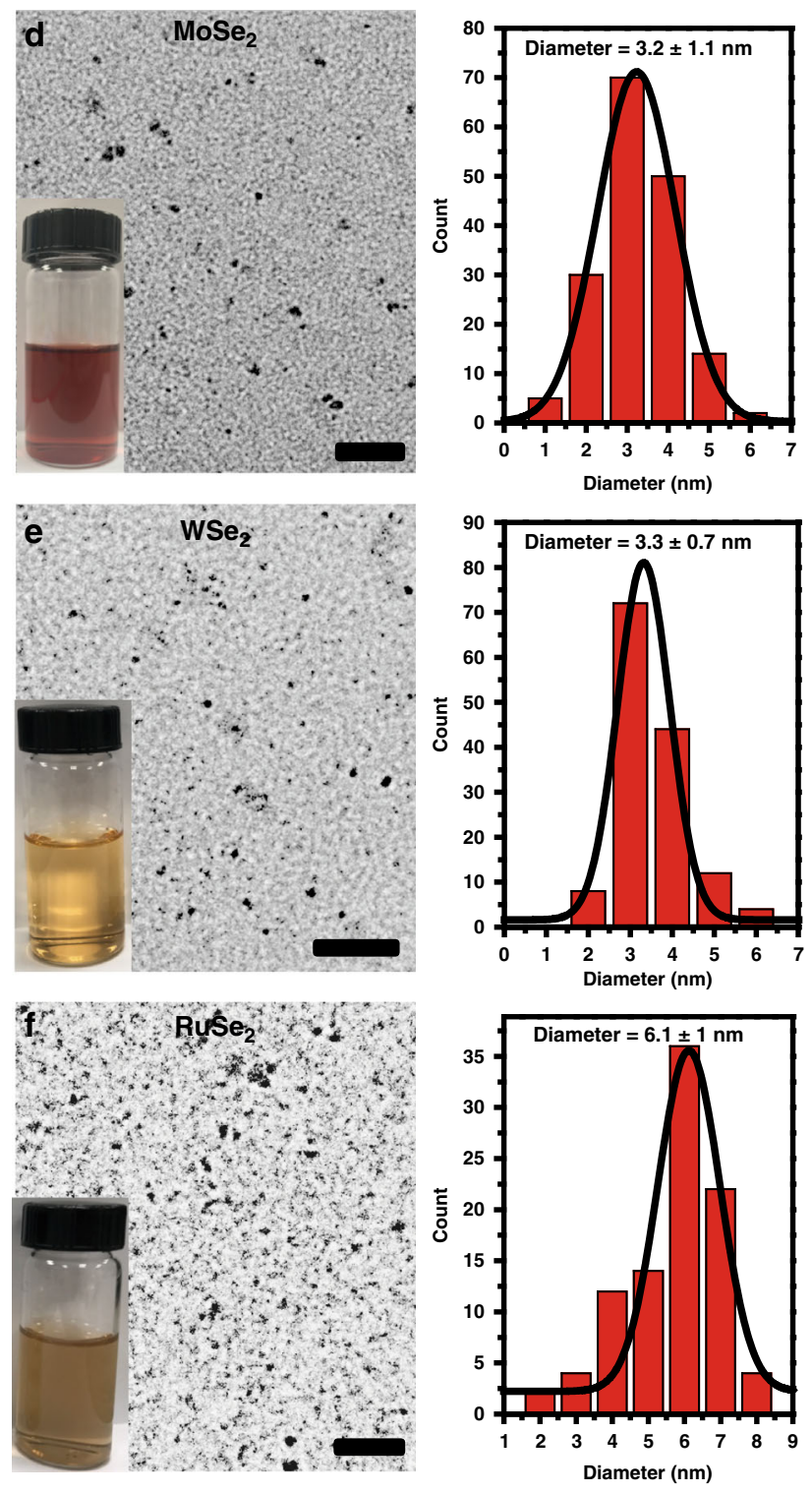

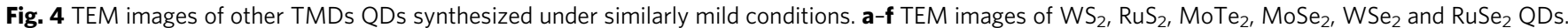
Insets: Stable colloidal suspension appearance of TMDs dispersed in aqueous condition. TMDs QDs have consistent sizes. Scale bar: 100 nm

The controversy of whether defect sites and photoluminescence quantum yields are positively or negatively correlated is still ongoing. The influences of surface defects on photoluminescence quantum yields in smaller sized $\mathrm{MoS}_{2}$ quantum dots is still largely unknown. From our observations, the intrinsic state emission of the highest to lowest defect sites groups, $\mathrm{MoS}_{2}-\mathrm{D}_{\mathrm{H}}$ to $\mathrm{MoS}_{2}-\mathrm{D}_{\mathrm{L}}$ QDs still play the leading role in the $\mathrm{PL}$ emission and especially showed significantly higher efficiency in $\mathrm{MoS}_{2}-\mathrm{D}_{\mathrm{H}} \mathrm{QDs}$. One of the possible explanation is the passivation effect from oxygen atoms in the $\mathrm{MoS}_{2}$ crystal lattice. In the presence of oxygen, the PL of CdSe QDs could be enhanced by as much as a factor of 6 , resulting from the surface passivation by oxygen on nanocrystalline surfaces ${ }^{42}$. This phenomenon of oxygen atoms induced surface passivation of QDs has also been identified in many other semiconductor $\mathrm{QDs}^{43,44}$. The embedded oxygen atoms in the crystalline of $\mathrm{MoS}_{2}$ structures, presumably played two roles; first by creating sulfur distortion defects which support defect state emission, second by forming Mo-S-O bond on the crystalline surface, which passivate it thus enabling the intrinsic state emission enhancement. Preliminary experiment on the sulfur defect (in the form of Mo-O) reveal that the defect could increase PL intensity. However, substantial work such as ultrafast dynamics studies can to explore these photophysics of $\mathrm{MoS}_{2}$ QDs in greater detail.

To obtain more information about the intrinsic changes of surface states, high-resolution XPS can reveal the surface chemical environment of the $\mathrm{MoS}_{2}$ samples. Figure 5e shows two characteristic peaks located at $\sim 229.6$ and $\sim 232.8 \mathrm{eV}$ in three spectrums, which arose from the $\mathrm{Mo}^{4+} 3 \mathrm{~d}_{5 / 2}$ and $\mathrm{Mo}^{4+} 3 d_{3 / 2}$, suggesting the dominance of Mo (IV) in the $\mathrm{MoS}_{2}$ samples. Compared with $\mathrm{MoS}_{2}-\mathrm{D}_{\mathrm{L}}$, samples with less sulfur amount show significantly broadened peaks from $\mathrm{MoS}_{2}-\mathrm{D}_{\mathrm{M}}$ to $\mathrm{MoS}_{2}-\mathrm{D}_{\mathrm{H}}$, confirming a higher degree of disorder in $\mathrm{MoS}_{2}-\mathrm{D}_{\mathrm{H}}{ }^{45}$. In addition, both $\mathrm{Mo}^{4+} 3 \mathrm{~d}_{5 / 2}$ and $\mathrm{Mo}^{4+} 3 d_{3 / 2}$ doublet peaks in three samples shifted to lower binding energies with increasing sulfur amount, reflecting the reduction of $\mathrm{MoS}_{2}$. This behavior was consistent with decreasing intensity of $\mathrm{Mo}^{6+}$ peaks at $\sim 236.2 \mathrm{eV}$, suggesting the presence of $\mathrm{Mo}-\mathrm{O}$ bonds in $\mathrm{MoS}_{2}-\mathrm{D}_{\mathrm{H}}$ decreased when sulfur amount approach to stoichiometric $\mathrm{MoS}_{2}$. These results indicate 
a

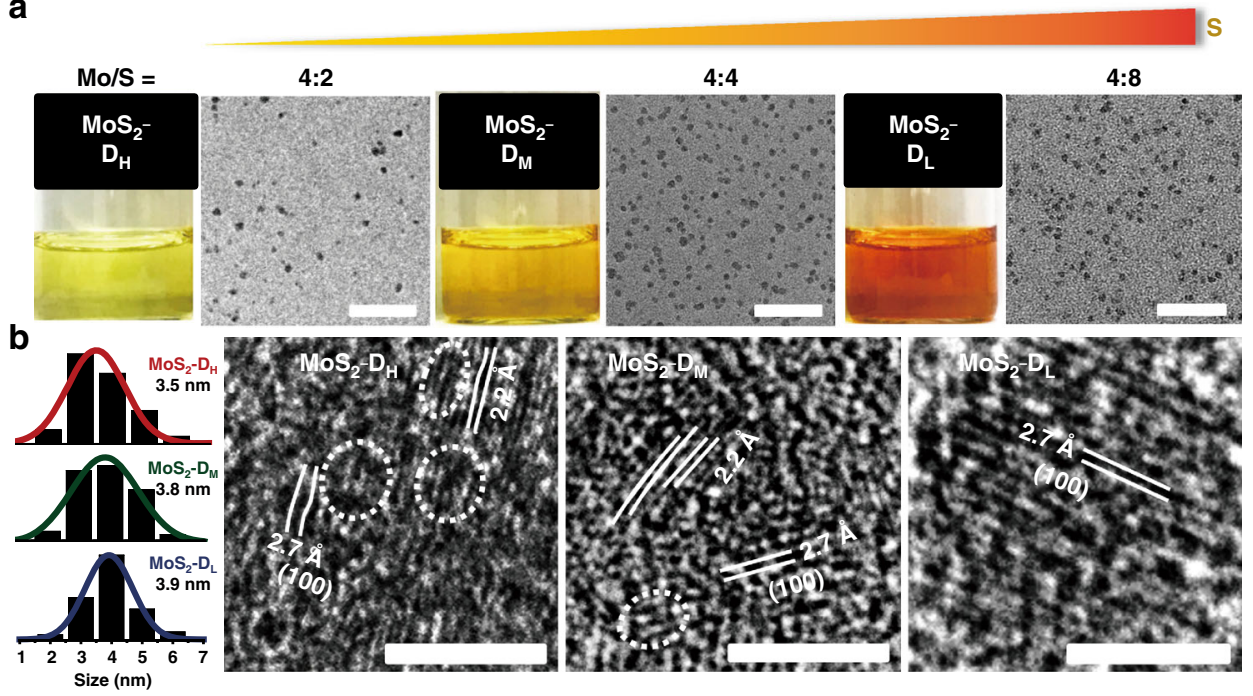

C

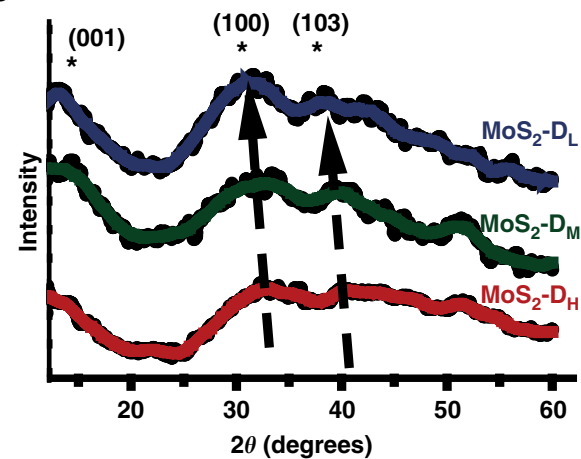

d

e
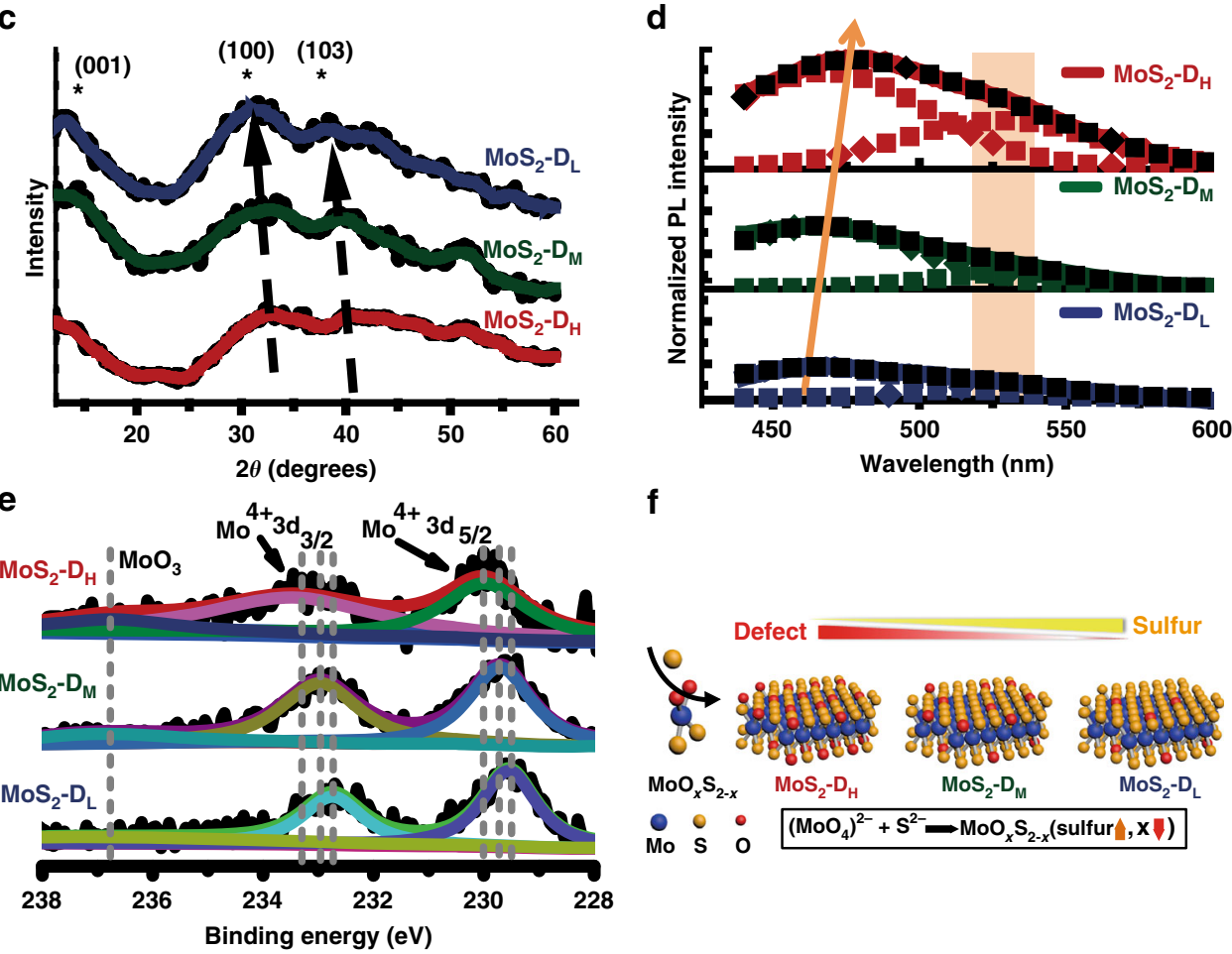

f

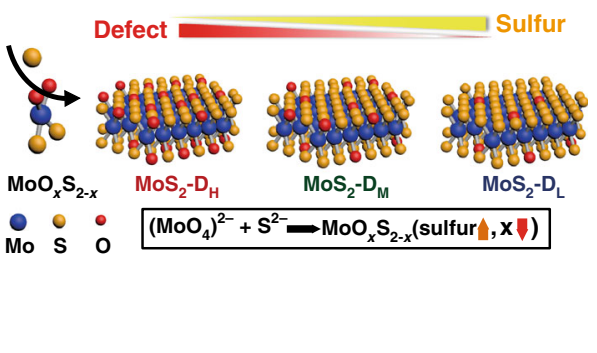

Fig. 5 Engineering sulfur defects into $\mathrm{MoS}_{2}$ QDs through stoichiometric reaction control. a Adjusting precursor ratios produces three kinds of MoS 2 suspension in pure water. TEM images show narrow size distribution. Scale bar: $50 \mathrm{~nm}$. b Different stoichiometry does not affect overall size of QD MoS 2 . (Distributions derived from at least $n=200$ ). HRTEM images show dislocations and distortions of lattice planes in MoS 2 QDs due to intrinsic defects. Scale bar: $2 \mathrm{~nm}$. c XRD show gradually enlarged lattice constants with decreasing stoichiometry in three $\mathrm{MoS}_{2}$ QDs. d PL spectrum show different emission intensities of the three $\mathrm{MoS}_{2}$ QDs at the same optical absorption. e XPS reveal the defect engineering was in the form of $\mathrm{MoO}_{x} \mathrm{~S}_{2-x}$ by the substitution of oxygen for sulfur. $\mathbf{f}$ Structural model of the reaction pathway of $\mathrm{MoS}_{2}$ QDs and defect engineering in $\mathrm{MoS}_{2}$ QDs as a function of precursor stoichiometry

that the defect engineering was in the form of $\mathrm{MoO}_{x} \mathrm{~S}_{2-x}$, by the substitution of oxygen for sulfur within the $\mathrm{MoS}_{2}$ lattices. This is also consistent with the XRD patterns that the substitution of the smaller $\mathrm{O}$ atom by the significantly larger $\mathrm{S}$ atom in the lattices induce the enlarged lattice constants (Fig. 5c). XPS quantification of the chemical compositions further revealed the atomic ratio of Mo to $S$ varied from 1:1.8 to 1: 2.34 (Supplementary Figure 13). So as sulfur amount increases $\left(\mathrm{MoS}_{2}-\mathrm{D}_{\mathrm{H}}\right.$ group to $\mathrm{MoS}_{2}-\mathrm{D}_{\mathrm{L}}$ group) resulted in the decreasing amount of oxygen atom in $\mathrm{MoO}_{x} \mathrm{~S}_{2}-{ }_{x}$, giving the direct evidence of sulfur amount-dependent degree of defects in $\mathrm{MoS}_{2}$ QDs.
Collectively the combined studies above, the reaction pathway of $\mathrm{MoS}_{2}$ QDs can be revealed and the defective crystal structures have been proved to obtain via a $\mathrm{MoO}_{4}^{2-}+\mathrm{S}^{2-} \rightarrow \mathrm{MoO}_{x} \mathrm{~S}_{2-x}$ pathway (Fig. 5f). The relatively lower sulfur amount was critically responsible for engineering the $\mathrm{MoS}_{2}$ QDs with S defects. With lower sulfur amount, the reaction became more insufficient, residual $\mathrm{Mo}-\mathrm{O}$ bonds (originally from $\mathrm{MoO}_{4}{ }^{2-}$ ) that remained embedded within the otherwise crystalline $\mathrm{MoS}_{2}$ structure; thus creating distortion defects in the observed structure. This reaction behavior is similar to previous observation of controlling synthesis temperature in preparing defective 
2D $\mathrm{MoS}_{2}$ nanosheets ${ }^{39}$. Here, $0 \mathrm{D} \mathrm{MoS}_{2}$ QDs with controllable defect engineering were realized by simply tuning the synthesis reagent stoichiometry at room temperature.

Surface vacancy associated singlet oxygen generation. While the influence of defects on 2D TMDs have been largely studied for tuning optical and physical properties, which widely lead to excellent electronic applications, until now the defect effect on TMDs QDs are suggestive and speculative based on extrapolation from 2D TMDs observations and not applied directly in engineerable defects driven bioapplications. In addition, compared to $2 \mathrm{D}$ materials, $0 \mathrm{D}$ nanomaterials were supposed to exhibit better biological behavior than their $2 \mathrm{D}$ counterparts ${ }^{46,47}$. Thus photochemical related ${ }^{1} \mathrm{O}_{2}$ generation capacity towards cancer therapy was investigated using our defect tunable $\mathrm{MoS}_{2}$ QDs. The singlet oxygen generation of the $\mathrm{MoS}_{2}$ was examined using 9, 10-anthracenediyl-bis(methylene) dimalonic acid (ABDA) as probe. It was found that without light irradiation, the absorbance of ABDA in three $\mathrm{MoS}_{2}$ QDs solution showed negligible decrease (Supplementary Figure 14). Under the white light irradiation, the absorption intensities within the groups decreased gradually with increased irradiation time (Fig. 6a). In addition, under the same irradiation condition without the presence of $\mathrm{MoS}_{2}$ QDs, both ${ }^{1} \mathrm{H}-\mathrm{NMR}$ and HPLC results did not show any appreciable change in the spectra of ABDA (Supplementary Figure 15), indicating the degradation of ABDA was indeed induced by the photosensitization from $\mathrm{MoS}_{2}$ QDs. The product of the ABDA trap after $\mathrm{MoS}_{2}$ QDs irradiation was checked by ${ }^{1} \mathrm{H}-\mathrm{NMR}$ and compared with the corresponding product of Rose Bengal (RB), a positive control known to generate ${ }^{1} \mathrm{O}_{2}$ under irradiation. Similar chemical shifts were also observed in the corresponding $\mathrm{H}$ peaks of the products after irradiation (Supplementary Figure 16), suggesting that the species generated from $\mathrm{MoS}_{2} \mathrm{QDs}$ and $\mathrm{RB}$ irradiation reacted with $A B D A$ and produced similar ABDA products. We checked again with repeating the irradiation of $\mathrm{MoS}_{2}$-ABDA reaction in $\mathrm{D}_{2} \mathrm{O}$ or $\mathrm{H}_{2} \mathrm{O}$ conditions. It was found that there was higher depletion of the ABDA substrate when using $\mathrm{D}_{2} \mathrm{O}$ while irradiating $\mathrm{MoS}_{2}$ QDs (Supplementary Figure 17). This showed that ${ }^{1} \mathrm{O}_{2}$ was generated from irradiation of $\mathrm{MoS}_{2}$ QDs. We further checked with a ${ }^{1} \mathrm{O}_{2}$ 2, 2, 6, 6-tetramethylpiperidine (TEMP) sensor-electron spin resonance spectrum (ESR) assay. We found more product after $5 \mathrm{~min}$ of $\mathrm{MoS}_{2}$ irradiation (Supplementary Figure 18). Collectively, ${ }^{1} \mathrm{O}_{2}$ was likely generated after $\mathrm{MoS}_{2}$ irradiation.

To compare the ${ }^{1} \mathrm{O}_{2}$ quantum yield of three MoS2 QDs upon light irradiation, calculation was performed based on the photochemical methods reported before ${ }^{48-50}$. The decreased $\mathrm{OD}$ at $400 \mathrm{~nm}\left(\mathrm{OD}_{400}\right)$ was performed to determine the decomposition rate constants of the photosensitizing process of three $\mathrm{MoS}_{2}$ QDs, which was obtained by fitting the various $\mathrm{OD}_{400}$ curves (Fig. 6b). By further integrating the optical absorption of $\mathrm{MoS}_{2}$ QDs in the range $400-800 \mathrm{~nm}$, the ${ }^{1} \mathrm{O}_{2}$ quantum yield of two kinds of $\mathrm{MoS}_{2}$ QDs relative to $\mathrm{MoS}_{2}-\mathrm{D}_{\mathrm{L}}$ were determined. The $\mathrm{MoS}_{2}-\mathrm{D}_{\mathrm{H}}$ and $\mathrm{MoS}_{2}-\mathrm{D}_{\mathrm{M}}$ groups respectively exhibited ${ }^{1} \mathrm{O}_{2}$ quantum yield of approximately 2.3 times and 1.7 times of $\mathrm{MoS}_{2}$ $\mathrm{D}_{\mathrm{L}}$ QDs' quantum yield (Fig. 6c, Supplementary Figure 19). Physical quenching between ${ }^{1} \mathrm{O}_{2}$ and N's lone pair electrons of amines may exist; especially those aromatic amines of the BSAsurfactant ${ }^{51}$. It is therefore important to check that the differences in ${ }^{1} \mathrm{O}_{2}$ quantum yield is not due to different amounts of BSA that are on the surface of the three kinds of $\mathrm{MoS}_{2}$ QDs. We quantified the amount of BSA on the surface of the three kinds of $\mathrm{MoS}_{2}$ using the micro-BCA protein assay. The BCA protein based assay showed no significantly different amounts of BSA on the same amount of the three $\mathrm{MoS}_{2}$ QDs defect types (Supplementary Figure 20). This indicated that the physical quenching due to proteins on the surface of the three $\mathrm{MoS}_{2}$ QDs groups are similar.
Thus, confirming that the significant increase in the ${ }^{1} \mathrm{O}_{2}$ quantum yields of $\mathrm{MoS}_{2}-\mathrm{D}_{\mathrm{M}}$ and $\mathrm{MoS}_{2}-\mathrm{D}_{\mathrm{H}}$ over $\mathrm{MoS}_{2}-\mathrm{D}_{\mathrm{L}}$ is due to increasing defects.

To further prove the correlations between the degree of defects and photodynamic efficiency, the ROS generation capacity was further detected inside the cancer cells by using dye dichlorohydrofluorescein diacetate (DCFH-DA) as an intracellular ROS indicator. DCFH-DA is non-fluorescent but can be oxidized by ROS to yield highly fluorescent-dichlorofluorescein (DCF). As shown in Fig. 6d, after irradiation, strongest green fluorescence was observed in $\mathrm{MoS}_{2}-\mathrm{D}_{\mathrm{H}}$ incubated groups, with relatively lower green fluorescence in $\mathrm{MoS}_{2}-\mathrm{D}_{\mathrm{M}}$ groups and the lowest green fluorescence in $\mathrm{MoS}_{2}-\mathrm{D}_{\mathrm{L}}$, which clearly confirmed the positive correlation between the degree of defects of $\mathrm{MoS}_{2}$ QDs and ROS generation capacity.

QDs acting as photosensitizers used in photodynamic therapy have attracted great attention in the area of nanomedicine ${ }^{52-57}$. In a typical photosensitizing process, the photosensitizer absorbs a quantum of light from ground state $\left(\mathrm{S}_{0}\right)$ to the excited singlet state $\left(\mathrm{S}_{1}\right)$, which is further converted to the excited triplet state $\left(\mathrm{T}_{1}\right)$ via intersystem crossing (ISC). The subsequent energy transferring from $\mathrm{T}_{1}$ to triplet oxygen $\left({ }^{3} \mathrm{O}_{2}\right)$ result in the formation of singlet oxygen $\left({ }^{1} \mathrm{O}_{2}\right)^{58}$. The quantum yield of ${ }^{1} \mathrm{O}_{2}$ generated by photosensitizers can be expressed as equation (1 $)^{59}$

$$
\phi_{\Delta}=\phi_{\mathrm{T}} \phi_{\mathrm{en}}
$$

where $\Phi_{\mathrm{T}}$ is the quantum yield of $\mathrm{T}_{1}$ formation and $\phi_{\mathrm{en}}$ is the efficiency of energy transfer from $\mathrm{T}_{1}$ to ${ }^{3} \mathrm{O}_{2}$. To understand the enhanced ROS generation mechanism in cells, we analyzed the relationship between the defects and corresponding ROS generation behavior in $\mathrm{MoS}_{2}$ QDs. Previous records have confirmed that $\Phi_{\mathrm{T}}$ is determinate by the ISC rate constants $k_{\mathrm{ISC}}$, which could be estimated from Eq. $(2)^{60}$

$$
k_{\mathrm{ISC}} \propto \frac{\left(\mathrm{T}_{1}\left|H_{\mathrm{SO}}\right| \mathrm{S}_{1}\right)^{2}}{\Delta E_{\mathrm{S} 1-\mathrm{T} 1}}
$$

Here $H_{\mathrm{SO}}$ represents the Hamiltonian for the spin-orbit perturbations and $\Delta E_{\mathrm{S} 1-\mathrm{T} 1}\left(\Delta E_{\mathrm{ST}}\right)$ is the energy gap between $\mathrm{S}_{1}$ and $\mathrm{T}_{1}$. Therefore by engineering the HOMO-LUMO energy level in molecules to reduce the energy gap $\Delta E_{\mathrm{ST}}$, high photosensitizing efficiency could be achieved ${ }^{61,62}$. In our $\mathrm{MoS}_{2}$ QDs experiments, we speculated that the sulfur vacancy derived defects engineered the bandgap of $\mathrm{MoS}_{2}$ QDs, thus modulating the $\Delta E_{\mathrm{ST}}$ to affect the photosensitizing capacity. To clarify this hypothesis, theoretical simulation using DFT was performed to check the density of states (DOS) of $\mathrm{MoS}_{2}$ QDs. After constructing a $3 \times 3 \times 1$ supercell structure, zero, one and two sulfur atoms in the supercell were removed and substituted by oxygen atoms (Fig. 6e). This leads to different degrees of sulfur defects in $\mathrm{MoS}_{2}$, which denoted as pristine $\mathrm{MoS}_{2}, \mathrm{MoS}_{2}-1 D_{0}$ and $\mathrm{MoS}_{2}-2 D_{0}$. The calculated DOS in Fig. $6 \mathrm{f}$ demonstrated that the vacancies of sulfur atoms and the substitution of oxygen atoms act as n-type dopants. The more substitution of more electronegative oxygen atoms led to more charge carriers, with narrower bandgap from pristine $\mathrm{MoS}_{2}$ of $1.68 \mathrm{eV}$ to $1.42 \mathrm{eV}\left(\mathrm{MoS}_{2}-1 D_{0}\right)$ and $1.30 \mathrm{eV}\left(\mathrm{MoS}_{2}-2 D_{0}\right)$. This decreasing bandgap of the simulated defective mode in $\mathrm{MoS}_{2}$ QDs qualitatively agrees with experimental observations from optical band gap (Supplementary Figure 21). Our calculation indicate that the sulfur defects in $\mathrm{MoS}_{2}$ QDs tend to reduce the bandgap with lowering $\Delta E_{\mathrm{ST}}$, which improved ISC efficiency and account for the observed enhanced ${ }^{1} \mathrm{O}_{2}$ generation in the photosensitizing process. 
a

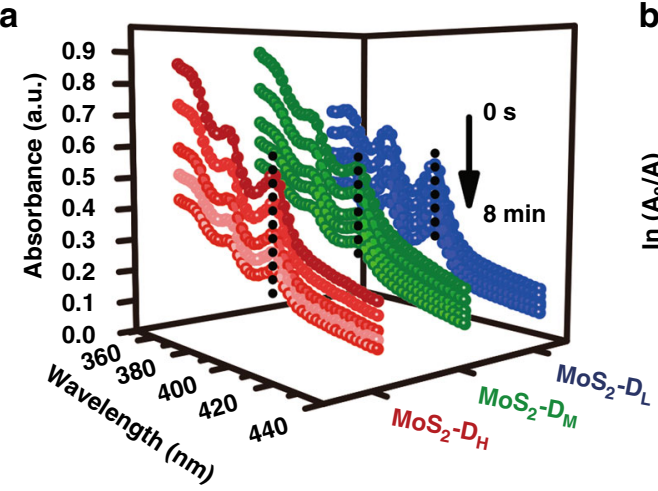

b

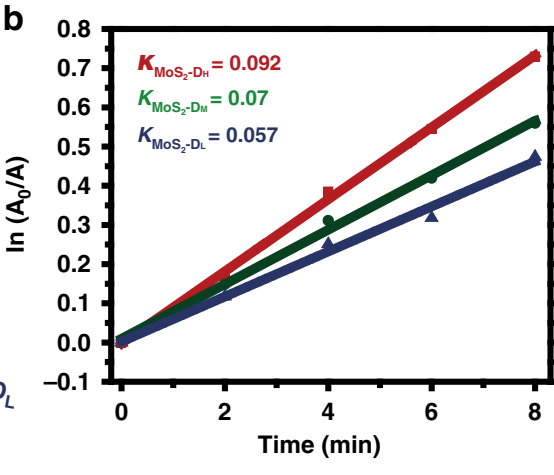

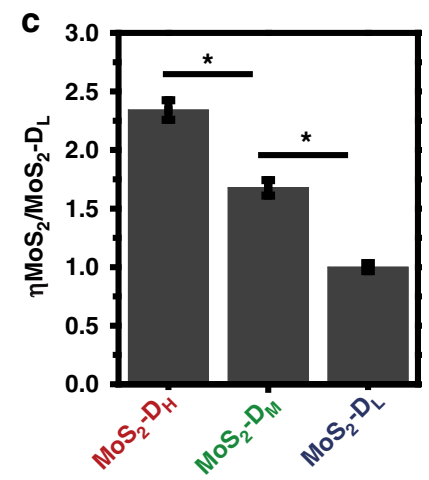

d

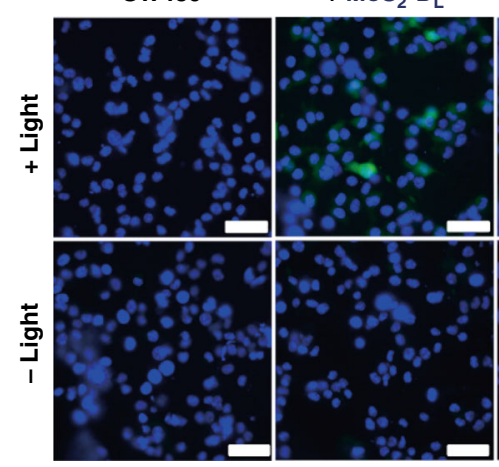

f

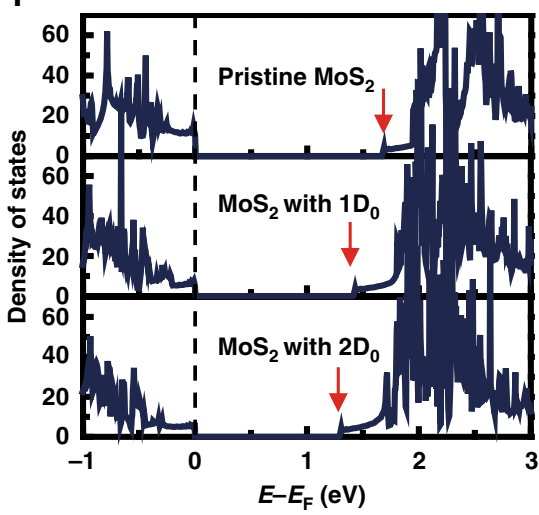

$+\operatorname{MoS}_{2}-D_{M}$

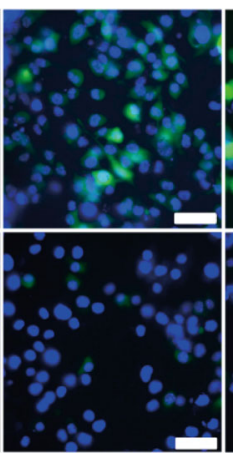

$+\mathrm{MoS}_{2}-\mathrm{D}_{\mathrm{H}}$

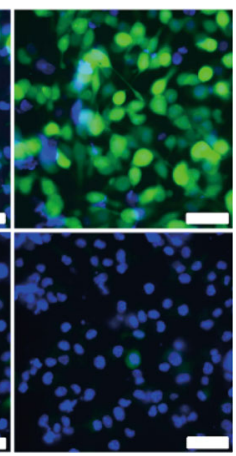

e

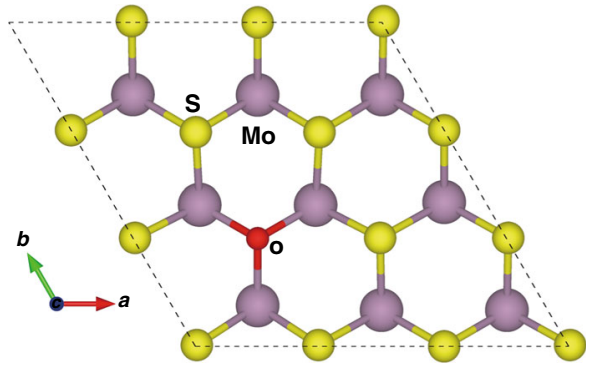

h

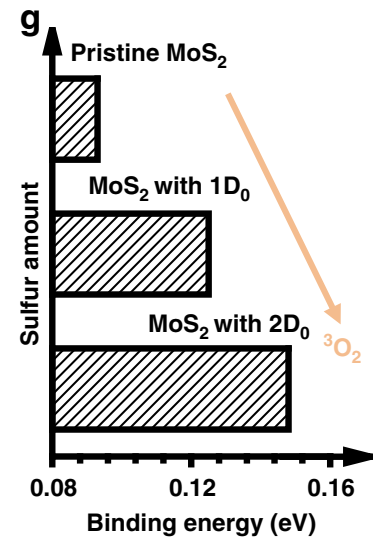

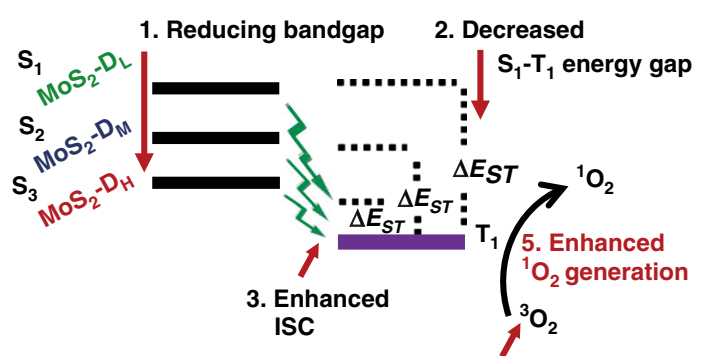

4. Higher affinity

$\mathrm{S}_{0}$

Fig. 6 Positive correlation between sulfur defects and photodynamic efficiency in QDs. a Absorption spectra of three kinds of MoS 2 QDs in the presence of ABDA under light irradiation $(0.1 \mathrm{~W} \mathrm{~cm}-2,8 \mathrm{~min})$. $\mathbf{b}$ Typical decomposition rate of the photosensitizing process, where $A_{0}$ is the absorbance of initial absorbance of $A B D A$ and $A$ is the absorbance of $A B D A$ under light irradiation at different time points (Full data plots can be found as Supplementary Figure 19). c Relative ${ }^{1} \mathrm{O}_{2}$ quantum yield of two $\mathrm{MoS}_{2}$ QDs groups relative to $\mathrm{MoS}_{2}-\mathrm{D}_{\mathrm{L}}$ QDs. Mean $\pm \mathrm{SD}, n=4$, Student's $t$-test, $p^{\star}<0.05$. $\mathbf{d}$ Detection of ROS generation at cellular level in colon cancer cell line, SW480 by intracellular ROS indicator $\mathrm{H}_{2}$ DCFH-DA. Scale bar: $50 \mu \mathrm{m}$. e Structure illustration for the substitution of oxygen for sulfur within $\mathrm{MoS}_{2}$ lattices. $\mathbf{f}$ Calculated density of states of $\mathrm{MoS}_{2}$ QDs show the defects reduce the bandgap. $\mathbf{g}$ More sulfur defects induce stronger binding affinity of ${ }^{3} \mathrm{O}_{2}$ to $\mathrm{MoS}_{2}$. h Proposed defect related ${ }^{1} \mathrm{O}_{2}$ generation mechanism of $\mathrm{MoS}_{2}$ QDs

On the other hand, the substitution of sulfur atom with the more electronegative oxygen atom modulated the charge density distributions in $\mathrm{MoS}_{2}$ crystals, affecting the Gibbs free energy for ${ }^{3} \mathrm{O}_{2}$ adsorption. To understand the binding affinity after different degrees of defect, the binding energies of ${ }^{3} \mathrm{O}_{2}$ on three $\mathrm{MoS}_{2}$ QDs were calculated by DFT. As shown in Fig. 6g, the binding energies for oxygen adsorption on $\mathrm{MoS}_{2}$ QDs decrease with less sulfur defect. The binding energies of ${ }^{3} \mathrm{O}_{2}$ on $\mathrm{MoS}_{2}-2 D_{0}$ are $0.15 \mathrm{eV}$, which are much larger than $\mathrm{MoS}_{2}-1 D_{0}(0.13 \mathrm{eV})$ and pristine $\mathrm{MoS}_{2}(0.09 \mathrm{eV})$. Moreover, with three sulfur atoms substituted by oxygen atoms, binding energy further increased to $0.17 \mathrm{eV}$. This result suggested that a higher defect $\mathrm{MoS}_{2}$ QD possessed a stronger binding affinity toward ${ }^{3} \mathrm{O}_{2}$ adsorption, which may allow higher oxygen coverage on the surface of $\mathrm{MoS}_{2} \mathrm{QD}$ in the energy transfer process, thus paving the way for higher $\phi_{\text {en }}$ in ${ }^{1} \mathrm{O}_{2}$ generation.

Besides the reduced bandgap and the strengthened binding affinity between $\mathrm{MoS}_{2}$ and ${ }^{3} \mathrm{O}_{2}$ (Fig. 6h), the spin-orbit perturbations $\left(H_{\mathrm{SO}}\right)$ in defective $\mathrm{MoS}_{2}$ QDs could also affect the intersystem crossing in the photosensitizing process. The vibronic coupling involved in Mo-S and Mo-O bonds would be significantly increased due to the increasing degree of defects. The decrease in size increases edge sites and dangling bonds in $\mathrm{MoS}_{2}$ QDs. This further enhances the likelihood of vibrational modes 
a
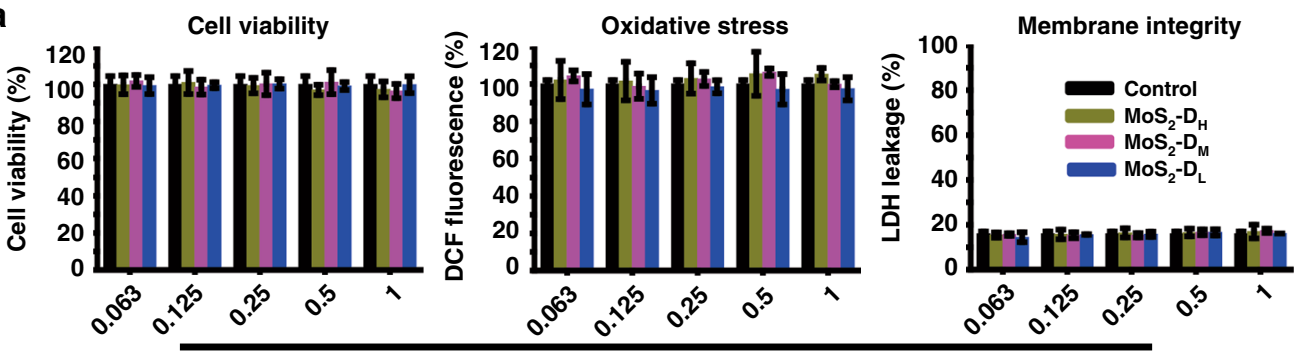

Concentration (mM)

b

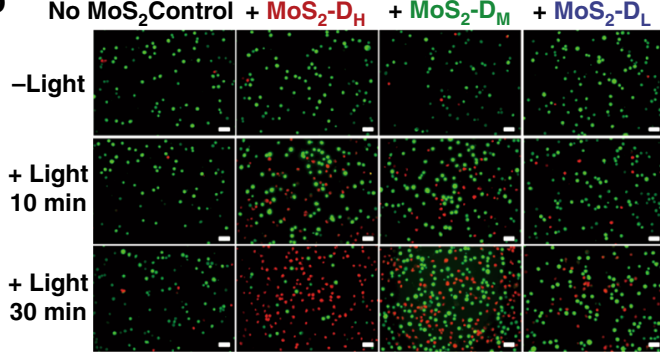

d

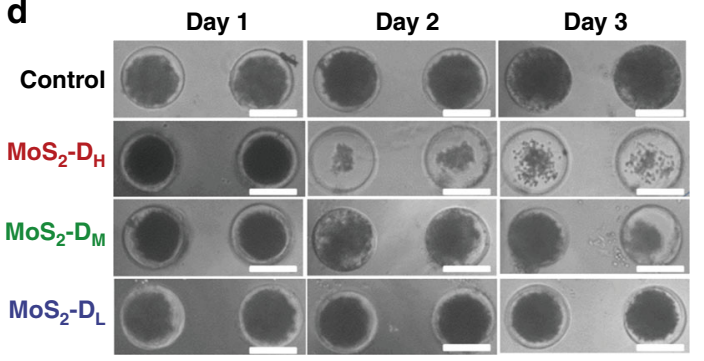

C

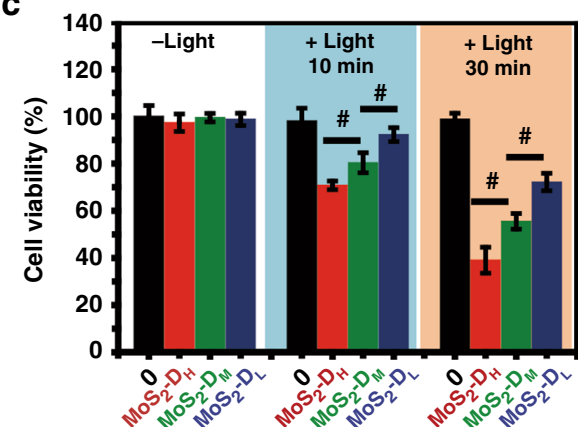

e

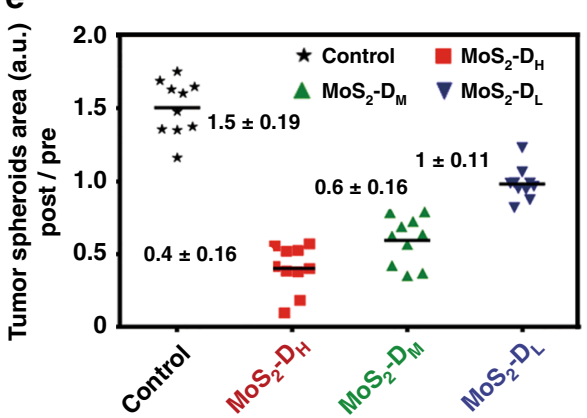

Fig. $7 \mathrm{MoS}_{2}$ QDs with more $S$ defects produce more oxidative stress in cancer cells. a MoS 2 QDs treatment (without light) on human endothelial cells (HMVEC) showed negligible cytotoxicity, negligible excessive ROS generation and low apoptosis induction. The measurements were performed in triplicate. $\mathbf{b}$ Calcein AM and PI co-staining and $\mathbf{c}$ cell viabilities of SW480 cells incubated with different defect laden MoS 2 QDs under white light exposure. The measurements were performed in triplicate. $\mathbf{d ~ M o S} 2-D_{H}$ showed the highest size reduction of 3D tumor spheroids assay. This implied high penetration of QDs into the interior of the 3D tumor spheroids mass with the highest defect group producing the highest oxidative stress that killed the cells. e Quantification of 3D tumor spheroids area post treatment and laser excitation with $\mathrm{MoS}_{2}-\mathrm{D}_{\mathrm{H}}$ showing the greatest cell death amongst the three MoS $\mathrm{S}_{2}$ groups. Mean $\pm S D, n=10$, Student's $t$ test, $p^{\#}<0.05$. Scale bar: $100 \mu \mathrm{m}$

hence promoting the intersystem crossing. The real exciton splitting situation due to the presence of defects and quantum confinement effect of typical semiconductor nanocrystals behavior in $\mathrm{MoS}_{2}$ QDs' case complicated their energy state. Here the proposed singlet and triplet states of $\mathrm{MoS}_{2}$ QD structure models was under the consideration of surface molecular states of $\mathrm{MoS}_{2}$ QDs. Further investigations to understand the electronic structure and exciton of $\mathrm{MoS}_{2}$ QDs are warranted.

Defect-dependent photodynamic therapy to kill cancer cells. Inspired by defect-dependent ROS generation capacity, the photodynamic efficiency of the three $\mathrm{MoS}_{2}$ QDs for killing cancer cells were further conducted. The material biocompatibility was first investigated. Cell viability, $\mathrm{LDH}$ and ROS level were examined to systematically evaluate the cytotoxicity effect (biocompatibility) of three $\mathrm{MoS}_{2}$ QDs using endothelial cells as a model for a non-cancer cell type which would be in contact with introduced $\mathrm{MoS}_{2}$ QDs bioapplications. Fig. 7a suggest that no obvious toxicity of all the three $\mathrm{MoS}_{2}$ QDs on human microvascular endothelial cell (HMVEC) cells at $\mathrm{MoS}_{2}$ QDs concentration as high as $1 \mathrm{mM}$ for $24 \mathrm{~h}$, suggesting good biocompatibility of all three $\mathrm{MoS}_{2}$ QDs types. There was no additional upregulation of ROS as the $\mathrm{MoS}_{2}$ QDs was not irradiated with light. When increasing the dose of $\mathrm{MoS}_{2} \mathrm{QDs}$, there was no increasing $\mathrm{LDH}$ leakage due to loss of membrane integrity as a result of induced apoptosis. We next used $\mathrm{MoS}_{2}$ QDs as photosensitizers in photodynamic cancer cell therapy in an in vitro model. Using SW480 cells as model cancer cells, Calcein AM (green fluorescence) and propidium iodide (PI, red fluorescence) staining were performed to visualize live/dead cells in the cancer cell therapy process. Control cell group with light treatments only or $\mathrm{MoS}_{2}$ QDs incubation only (without light treatment) maintained high cell viability (Fig. $7 \mathrm{~b}$ ). As expected, cells incubated with higher defects $\mathrm{MoS}_{2}-\mathrm{D}_{\mathrm{H}}$ QDs showed considerably lower cell viability under light irradiation compared to $\mathrm{MoS}_{2}-\mathrm{D}_{\mathrm{M}}$ and $\mathrm{MoS}_{2}-\mathrm{D}_{\mathrm{L}}$ treatment groups (Fig. 7c). After being irradiated for a short $10 \mathrm{~min}$, only $72 \%$ of cells incubated with $\mathrm{MoS}_{2}-\mathrm{D}_{\mathrm{H}}$ were viable, which further decreased to $39.7 \%$ when the irradiation 
time prolonged to $30 \mathrm{~min}$. At the same treatment concentrations, cell viability of $\mathrm{MoS}_{2}-\mathrm{D}_{\mathrm{M}}$ and $\mathrm{MoS}_{2}-\mathrm{D}_{\mathrm{L}}$ groups decreased from $81 \%$ to 55.2 and $92 \%$ to $72 \%$ respectively, as the irradiation time was prolonged from $10 \mathrm{~min}$ to $30 \mathrm{~min}$.

These results demonstrated that $\mathrm{MoS}_{2}$ QDs with higher defects are more effective photodynamic agents to kill cancer cells over those with lower defects at the same concentration.

To further test the photodynamic efficiency in vitro on cancer cells, 3D SW480 colorectal tumor spheroids were used as a more realistic cancer model to simulate the therapeutic responses of three $\mathrm{MoS}_{2}$ QDs groups ${ }^{63,64}$. To examine the photodynamic effect, 3D tumor spheroids were incubated with different $\mathrm{MoS}_{2}$ QDs groups for $6 \mathrm{~h}$ to allow for increased penetration and then irradiated with white light for $30 \mathrm{~min}$. After three days, as expected, the size of tumor spheroids incubated with $\mathrm{MoS}_{2}$ QDs with a single irradiation show higher degree of growth retardation compared to control group without $\mathrm{MoS}_{2}$ treatment (Fig. 7d, e, Supplementary Figure 22). More importantly, the $\mathrm{MoS}_{2}-\mathrm{D}_{\mathrm{H}}$ treated groups show the greatest tumor spheroids size reduction, as compared with that of the $\mathrm{MoS}_{2}-\mathrm{D}_{\mathrm{M}}$ and $\mathrm{MoS}_{2}-\mathrm{D}_{\mathrm{L}}$ groups at equivalent $\mathrm{MoS}_{2}$ concentrations. These results indicated that the degree of defects was related to the photodynamic efficiency of $\mathrm{MoS}_{2}$ QDs, showing the possible defect engineering in QDs to enhance their therapy effect while reducing their actual dosage used in clinic, thereby preventing the potential chronic side effects and complications.

\section{Discussion}

In summary, we have demonstrated the biomineralization assisted bottom-up strategy for synthesizing a wide range of QDs from the TMD family with using mild conditions. The strategy employed straightforward chemical reactions between sodium chalcogenides and transition metal chlorides or transition metal oxides with a high yield reaction within a matter of tens of seconds. Disulfide bonds were shown to be the functional group for size quality control, and further verified by DFT simulations. The major benefit of the synthetic route is the ability of controllable defects engineering on TMD QDs via tuning precursor stoichiometry. Our studies revealed that the reaction pathway of $\mathrm{MoS}_{2}$ QDs and the defective crystal structures might be $\left(\mathrm{MoO}_{4}\right)^{2-}+\mathrm{S}^{2-} \rightarrow \mathrm{MoO}_{x} \mathrm{~S}_{2-x}$. This synthetic strategy simplifies the synthetic process with mild conditions, enriching the TMD QDs library for exploring their physical and chemical properties and related applications. Our bottom-up strategy for synthesizing TMDs QDs provided an ideal platform for investigating the correlation between the surface defects of semiconductor nanomaterials and the corresponding capacity of anti-cancer oxidative stress generation. Strongly positive correlation between degrees of sulfur defects and photodynamic efficiencies could be observed in the prepared $\mathrm{MoS}_{2}$ QDs. The density of states and molecular dynamics calculations suggest that the sulfur defect in $\mathrm{MoS}_{2}$ QDs reduced the bandgap and strengthened the binding affinity between $\mathrm{MoS}_{2}$ QDs and ${ }^{3} \mathrm{O}_{2}$. This may have contributed to the intersystem crossing and energy transfer separately in the photosensitizing process; highlighting the significant potential for defect engineering as an intrinsic alteration tool used in conjunction with existing TMDs bionanotechnologies ${ }^{65-67}$ without adding yet another material.

\footnotetext{
Methods

Materials. All chemicals and reagents were used as received without any further purification. $\mathrm{MoCl}_{5}(95 \%), \mathrm{MoO}_{3}(95 \%)$ and Bovine Serum Album (BSA) were purchased from Sigma-Aldrich Co. (USA). Deionized water was used throughout the synthesis.
}

Sample characterization. The morphologies and size of $\mathrm{MoS}_{2}$ QDs samples were characterized by TEM (FE-TEM; JEOL JEM-2100F, Japan). Nanoparticle sizes were determined by measuring no $<200$ randomly selected nanoparticles from TEM micrographs with ImageJ (http: //rsbweb.nib.gov/ij/). The powder XRD measurements were performed using a Bruker D8 advanced diffractometer with a $\mathrm{Cu} \mathrm{Ka}$ irradiation in the $2 \theta$ range of $20^{\circ}-60^{\circ}$. The elemental composition and binding energy of the sample were characterized by X-ray photoelectron spectroscopy (XPS; AXIS HIS, Kratos Analytical). The absorbance spectrum scanning and fluorescence intensities were conducted using microplate reader (BioTEK H4FM, USA). Fluorescence imaging were taken with inverted fluorescence microscope (Leica DMI6000, Germany) and the phase contrast images were captured by inverted microscope (Olympus-CX41, Japan).

Synthesis of $\mathbf{M o S}_{\mathbf{2}}$ quantum dots. In a typical procedure, Mo-precursor solution was firstly prepared by dissolving $\mathrm{MoCl}_{5}$ or $\mathrm{MoO}_{3}$ into $\mathrm{dH}_{2} \mathrm{O}$ by adjusting the $\mathrm{pH}$ value to be 11 . The resultant solution was transparent and colorless after sonication. Then $1 \mathrm{~mL}$ of Mo precursor solution mixed into $39 \mathrm{~mL}$ of BSA solution $(1 \mathrm{mg} / \mathrm{mL})$ followed by adding $0.2 \mathrm{~mL} 0.5 \mathrm{M} \mathrm{Na}_{2} \mathrm{~S}$ solution under vigorous stirring at room temperature. The $\mathrm{pH}$ value of the mixed solution was subsequently adjusted to $6 \sim 7$ by adding $1 \mathrm{M} \mathrm{HCl}$. After neutralizing the $\mathrm{pH}$, a clear yellow solution of $\mathrm{MoS}_{2}$ quantum dots was produced quickly.

Synthesis of other TMD quantum dots with BSA. The protocol of synthesizing other TMD quantum dots was similar to $\mathrm{MoS}_{2}$ quantum dots. The only difference was the preparation of $\mathrm{Se}$ - and $\mathrm{Te}$ - precursors. In a typical preparation of $\mathrm{Se}$ precursor, $79.9 \mathrm{mg}$ of Se powder was added into $1 \mathrm{~mL}$ of $\mathrm{NaBH}_{4}$ solution $(80 \mathrm{mg})$ to reduce it at ambient condition. 30 min later, black Se powder disappeared completely and the clear solution was Se precursor. For the preparation of Te precursor, $31.9 \mathrm{mg}$ of Te powder was added into $200 \mu \mathrm{L} \mathrm{NaBH}$ solution (28.4 mg). After $30 \mathrm{~min}$, black Te powder fully disappeared and the clear solution was $\mathrm{Te}$ precursor. For the synthesis of $\mathrm{WS}_{2}, \mathrm{RuS}_{2}, \mathrm{MoTe}_{2}, \mathrm{WSe}_{2}$ and $\mathrm{RuSe}_{2}$, similarly mild conditions were carried as $\mathrm{MoS}_{2}$ above.

Photodynamic evaluation of three $\mathrm{MoS}_{\mathbf{2}}$ QDs with ABDA. In the experiments ABDA as the probe of ${ }^{1} \mathrm{O}_{2}$ was added to different $\mathrm{MoS}_{2}$ QDs dispersion solution with final concentration of $10 \mu \mathrm{M}$. Then the mixture solution was transferred to a cuvette and exposed to white light $(400-800 \mathrm{~nm}, 100 \mathrm{~mW})$ perpendicularly for $8 \mathrm{~min}$. The light source irradiated a region of $1 \mathrm{~cm}^{2}$. For the convenience of observation, the OD of three $\mathrm{MoS}_{2}$ QDs at $400 \mathrm{~nm}$ was adjusted to the same. The absorbance change at $400 \mathrm{~nm}$ was recorded at various time points to obtain the degradation rate of ABDA. For the comparison of ${ }^{1} \mathrm{O}_{2}$ quantum yield, all the ${ }^{1} \mathrm{O}_{2}$ quantum yield of $\mathrm{MoS}_{2}$ was normalized to that of $\mathrm{MoS}_{2}-\mathrm{D}_{\mathrm{L}}$, relative ${ }^{1} \mathrm{O}_{2} \eta$ to $\mathrm{MoS}_{2}-\mathrm{D}_{\mathrm{L}}$ using the following equation:

$$
\frac{\mathrm{MoS}_{2}}{\mathrm{MoS}_{2}-\mathrm{D}_{\mathrm{L}}}=\frac{K_{\mathrm{MoS}_{2}} A_{\mathrm{MoS}_{2}-\mathrm{D}_{\mathrm{L}}}}{K_{\mathrm{MoS}_{2}-\mathrm{D}_{\mathrm{L}}} A_{\mathrm{MoS}_{2}}},
$$

where $K_{\mathrm{MoS}}$ and $K_{\mathrm{MoS}_{2}-\mathrm{D}_{\mathrm{L}}}$ represent the decomposition rate constants of the photodegradation of ABDA with different $\mathrm{MoS}_{2}$, which were determined by plotting $\log _{e}\left(\mathrm{Abs}_{0} / \mathrm{Abs}\right)$ versus irradiation, where $\mathrm{Abs}_{0}$ is the initial absorbance of $\mathrm{ABDA}, \mathrm{Abs}$ is the ABDA absorbance at different irradiation time. $A_{\mathrm{MoS}_{2}}$ and $A_{\mathrm{MoS}}-\mathrm{D}_{\mathrm{L}}$ refer to the light absorbed by different $\mathrm{MoS}_{2}$ and $\mathrm{MoS}_{2}-\mathrm{D}_{\mathrm{L}}$ respectively, which are determined by integration of the optical absorption bands in the wavelength range from 400 to $800 \mathrm{~nm}$.

Intracellular ROS assay. DCF-DA probed the ROS generation inside cells. SW480 cells were cultured on 8-well cell chamber slides overnight. Overnight medium was replaced with fresh medium containing different $\mathrm{MoS}_{2}$ QDs (at $0.5 \mathrm{mM}$ ). After incubation for $6 \mathrm{~h}$, DCF-DA $(25 \mu \mathrm{M})$ was added for $15 \mathrm{~min}$, and then either left in the dark or irradiation with white light $\left(100 \mathrm{~mW} \mathrm{~cm}^{-2}\right)$ for $5 \mathrm{~min}$. After washing and staining the cells with Hoechst $33342(5 \mu \mathrm{g} / \mathrm{mL})$, fluorescence images of the cells were captured using Nikon A1 confocal microscope (Nikon, Japan).

Photodynamic killing of SW480 cancer cells. To assess the photodynamic effect of $\mathrm{MoS}_{2}$ QDs on SW480 cells, SW480 cells was seeded and cultured in 96-well plates overnight. Then different groups of $\mathrm{MoS}_{2}$ QDs were used to treat SW480 cells for $6 \mathrm{~h}$. Subsequently, cells were irradiated with white light $\left(100 \mathrm{~mW} \mathrm{~cm}^{-2}\right)$ for 10 and $30 \mathrm{~min}$. After treatments, the cells were further cultured for $12 \mathrm{~h}$. Cell viability after different treatments was evaluated by WST assays and Tali Image based Cytometer (Life Technologies, USA).

Formation and photodynamic efficiency in SW480 colorectal tumor spheroids. To examine the photodynamic effect on 3D SW480 colorectal tumor spheroids, 3D cell spheroids were first prepared. The solidified agrose micro-molds were sterilized by UV irradiation for $30 \mathrm{~min}$ and then equilibrated with DMEM medium for $12 \mathrm{~h}$. After that $50 \mu \mathrm{L}$ of SW480 cell suspension were seeded into each agarose micro-mold, $5 \mathrm{~min}$ after the cells settle down into the micro-mold, $500 \mu \mathrm{L}$ of DMEM medium was added to the well. After $24 \mathrm{~h}, 3 \mathrm{D}$ SW480 cell spheroids were 
formed due to gravity and the aggregation of cells. For photodynamic therapy treatment, the different $\mathrm{MoS}_{2}$ QDs were added into 3D SW480 cell spheroids. After $6 \mathrm{~h}, 3 \mathrm{D}$ SW480 cell spheroids were either exposed to dark or white light $\left(100 \mathrm{~mW} \mathrm{~cm}^{-2}\right)$ for $30 \mathrm{~min}$. The images of 3D SW480 cell spheroids were captured by a light microscope (Olympus-CX41, Japan) in the subsequent days and the size of the 3D SW480 cell spheroids were measured with ImageJ software.

\section{Density functional theory (DFT) binding energy siumlation. Adsorptions of} various chemical groups on $\mathrm{MoS}_{2}$ were investigated by performing simulations at the density functional theory (DFT) level as implemented in the Vienna ab initio simulation package (VASP) ${ }^{68}$, with the exchange-correlation functional of PerdewBurke-Ernzerhof $(\mathrm{PBE})^{69}$. The long-range van der Waals interactions were calculated within the Tkatchenko and Scheffler scheme to avoid the empirical parameters ${ }^{70}$, while the self-consistent screening and polarizability contraction effects were also taken into account, in view of their important roles in determining the weak inter-molecular interactions ${ }^{71}$. The $\mathrm{MoS}_{2}$ layer was modeled with a vacuum region of $20 \AA$. The first Brillouin zone was sampled with a $k$-point mesh with a plane-wave cutoff of $450 \mathrm{eV}$. The various molecules including $\mathrm{CH}_{3}-\mathrm{X}(\mathrm{X}=-\mathrm{OH}$, $-\mathrm{SH},-\mathrm{NH}_{2},-\mathrm{COOH},-\mathrm{SSCH}_{3}$ and $\left.-\mathrm{C}_{6} \mathrm{H}_{5}\right)$ and $\mathrm{CH}_{3}-\mathrm{CONH}-\mathrm{CH}_{3}$ were simulated following the equation $E_{\text {ad }}=E_{\mathrm{MoS}_{2}+\text { group }}-E_{\text {group }}-E_{\mathrm{MoS}_{2}}$ with the results summarized in Supplementary Table 1 .

\section{Methodology for density of states (DOS) of models $\mathrm{MoS}_{2}$}

- DFT based VASP

- PAW-PBE potential electron-ion interaction and exchange-correlation.

- $3 \times 3 \times 1$ supercell structure is applied

- $500 \mathrm{eV}$ for plane-wave expansion cutoff

- $7 \times 7 \times 1$ Gama-centred k-point mesh

- All structures were relaxed until the force is smaller than $0.001 \mathrm{eV} /$ Angstrom with a total energy convergence criterion of $1 \times 10^{-6} \mathrm{eV}$.

- Vacuum is $15 \AA$.

- The relaxed lattice parameter for $\mathrm{MoS}_{2}$ monolayer is $3.190 \AA$

\section{Data availability}

All the relevant data are available from the corresponding authors upon reasonable request.

Received: 8 June 2018 Accepted: 22 November 2018

Published online: 03 January 2019

\section{References}

1. Chhowalla, M. et al. The chemistry of two-dimensional layered transition metal dichalcogenide nanosheets. Nat. Chem. 5, 263 (2013).

2. Duan, X., Wang, C., Pan, A., Yu, R. \& Duan, X. Two-dimensional transition metal dichalcogenides as atomically thin semiconductors: opportunities and challenges. Chem. Soc. Rev. 44, 8859-8876 (2015).

3. Liu, B. et al. Synthesis and optimization of $\mathrm{MoS}_{2} @ \mathrm{Fe}_{3} \mathrm{O}_{4}-\mathrm{ICG} / \mathrm{Pt}$ (IV) nanoflowers for MR/IR/PA bioimaging and combined PTT/PDT/ chemotherapy triggered by $808 \mathrm{~nm}$ laser. Adv. Sci. 4, 1600540 (2017).

4. Zhu, X. et al. Intracellular mechanistic understanding of $2 \mathrm{D} \mathrm{MoS}$ nanosheets for anti-exocytosis-enhanced synergistic cancer therapy. ACS Nano 12, 2922-2938 (2018).

5. Yu, F. et al. Ultrasensitive pressure detection of few-layer $\mathrm{MoS}_{2}$. Adv. Mater. 29, 1603266 (2017)

6. Liu, K.-K. et al. Growth of large-area and highly crystalline MoS2 thin layers on insulating substrates. Nano. Lett. 12, 1538-1544 (2012).

7. Deng, D. et al. Catalysis with two-dimensional materials and their heterostructures. Nat. Nanotechnol. 11, 218 (2016).

8. Liu, C. et al. Rapid water disinfection using vertically aligned $\mathrm{MoS}_{2}$ nanofilms and visible light. Nat. Nanotechnol. 11, 1098 (2016).

9. Lopez-Sanchez, O., Lembke, D., Kayci, M., Radenovic, A. \& Kis, A. Ultrasensitive photodetectors based on monolayer $\mathrm{MoS}_{2}$. Nat. Nanotechnol. 8 , 497 (2013).

10. Bonaccorso, F. et al. Graphene, related two-dimensional crystals, and hybrid systems for energy conversion and storage. Science 347, 1246501 (2015).

11. Acerce, M., Voiry, D. \& Chhowalla, M. Metallic $1 T$ phase $\mathrm{MoS}_{2}$ nanosheets as supercapacitor electrode materials. Nat. Nanotechnol. 10, 313 (2015).

12. Neville, R. \& Evans, B. The band edge excitons in $2 \mathrm{H}-\mathrm{MoS}_{2}$. Phys. Status Solidi B. 73, 597-606 (1976).

13. Zhang, X. et al. A facile and universal top-down method for preparation of monodisperse transition-metal dichalcogenide nanodots. Angew. Chem., Int. Ed. 54, 5425-5428 (2015).
14. Tan, C. et al. Preparation of High-Percentage 1T-phase transition metal dichalcogenide nanodots for electrochemical hydrogen evolution. Adv. Mater. 30, 1705509 (2018).

15. Eda, G. et al. Photoluminescence from chemically exfoliated $\mathrm{MoS}_{2}$. Nano. Lett. 11, 5111-5116 (2011)

16. Coleman, J. N. et al. Two-dimensional nanosheets produced by liquid exfoliation of layered materials. Science 331, 568-571 (2011).

17. Zhou, J. et al. A library of atomically thin metal chalcogenides. Nature 556, 355 (2018).

18. Yong, Y. et al. Tungsten sulfide quantum dots as multifunctional nanotheranostics for in vivo dual-modal image-guided photothermal/ radiotherapy synergistic therapy. ACS Nano 9, 12451-12463 (2015).

19. Najafi, L. et al. Solution-processed hybrid graphene flake/2H-MoS 2 quantum dot hetero-structures for efficient electrochemical hydrogen evolution. Chem. Mater. 29, 5782-5786 (2017).

20. Wang, Xiaojie et al. One-step synthesis of water-soluble and highly fluorescent $\mathrm{MoS}_{2}$ quantum dots for detection of hydrogen peroxide and glucose. Sens. Actuat. B-Chem. 252, 183-190 (2017).

21. Mishra, Himanshu et al. $\mathrm{pH}$ dependent optical switching and fluorescence modulation of molybdenum sulfide quantum dots. Adv. Opt. Mater. 5, 1601021 (2017).

22. Zhang, Shuqu et al. $\mathrm{MoS}_{2}$ quantum dot growth induced by $\mathrm{s}$ vacancies in a znin2s4 monolayer: atomic-level heterostructure for photocatalytic hydrogen production. ACS Nano 12, 751-758 (2017).

23. Samia, A. C., Chen, X. \& Burda, C. Semiconductor quantum dots for photodynamic therapy. J. Am. Chem. Soc. 125, 15736-15737 (2003).

24. Wang, Z. et al. Biomineralization-inspired synthesis of copper sulfide-ferritin nanocages as cancer theranostics. ACS Nano 10, 3453-3460 (2016).

25. Dong, Z. et al. Synthesis of hollow biomineralized $\mathrm{CaCO}_{3}$-polydopamine nanoparticles for multimodal imaging-guided cancer photodynamic therapy with reduced skin photosensitivity. J. Am. Chem. Soc. 140, 2165-2178 (2018).

26. Yan, Y. et al. Facile synthesis of water-soluble $\mathrm{WS}_{2}$ quantum dots for turn-on fluorescent measurement of lipoic acid. J. Phys. Chem. C. 120, 12170-12177 (2016).

27. Baker, M., Gilmore, R., Lenardi, C. \& Gissler, W. XPS investigation of preferential sputtering of $\mathrm{S}$ from $\mathrm{MoS}_{2}$ and determination of MoSx stoichiometry from Mo and S peak positions. Appl. Surf. Sci. 150, 255-262 (1999).

28. Chikan, V. \& Kelley, D. Size-dependent spectroscopy of $\mathrm{MoS}_{2}$ nanoclusters. J. Phys. Chem. B 106, 3794-3804 (2002).

29. Sun, J. et al. Mechanistic understanding of excitation-correlated nonlinear optical properties in $\mathrm{MoS}_{2}$ nanosheets and nanodots: the role of exciton resonance. ACS Photonics 3, 2434-2444 (2016).

30. Doolen, R., Laitinen, R., Parsapour, F. \& Kelley, D. Trap state dynamics in $\mathrm{MoS}_{2}$ nanoclusters. J. Phys. Chem. B 102, 3906-3911 (1998).

31. Narayanan, K. L. et al. Effect of irradiation-induced disorder on the optical absorption spectra of CdS thin films. Phys. B: Condens. Matter 240, 8-12 (1997).

32. Mocatta, David et al. Heavily doped semiconductor nanocrystal quantum dots. Science 332, 77-81 (2011).

33. Jean, Joel et al. Radiative efficiency limit with band tailing exceeds $30 \%$ for quantum dot solar cells. ACS Energy Lett. 2, 2616-2624 (2017).

34. Liu, T. et al. Drug delivery with PEgylated $\mathrm{MoS}_{2}$ nano-sheets for combined photothermal and chemotherapy of cancer. Adv. Mater. 26, 3433-3440 (2014).

35. Guan, G. et al. Protein induces layer-by-layer exfoliation of transition metal dichalcogenides. J. Am. Chem. Soc. 137, 6152-6155 (2015).

36. Guan, G. et al. Convenient purification of gold clusters by co-precipitation for improved sensing of hydrogen peroxide, mercury ions and pesticides. Chem. Commun. 50, 5703-5705 (2014)

37. Sun, Y. et al. Low-temperature solution synthesis of few-layer $1 \mathrm{~T}^{\prime}-\mathrm{MoTe}_{2}$ nanostructures exhibiting lattice compression. Angew. Chem., Int. Ed. 55, 2830-2834 (2016).

38. Walsh, A. \& Zunger, A. Instilling defect tolerance in new compounds. Nat. Mater. 16, 964 (2017)

39. Xie, J. et al. Controllable disorder engineering in oxygen-incorporated $\mathrm{MoS}_{2}$ ultrathin nanosheets for efficient hydrogen evolution. J. Am. Chem. Soc. 135 17881-17888 (2013).

40. Zhu, S. et al. Surface chemistry routes to modulate the photoluminescence of graphene quantum dots: From fluorescence mechanism to up-conversion bioimaging applications. Adv. Funct. Mater. 22, 4732-4740 (2012).

41. Zhu, S. et al. Highly photoluminescent carbon dots for multicolor patterning, sensors, and bioimaging. Angew. Chem., Int. Ed. 125, 4045-4049 (2013).

42. Myung, Noseung, Yoonjung, Bae \& Allen, J. Bard Enhancement of the photoluminescence of CdSe nanocrystals dispersed in $\mathrm{CHCl}_{3}$ by oxygen passivation of surface states. Nano. Lett. 3, 747-749 (2003).

43. Jang, Eunjoo et al. Surface treatment to enhance the quantum efficiency of semiconductor nanocrystals. J. Phys. Chem. B 108, 4597-4600 (2004). 
44. Jung, Dae-Ryong et al. Semiconductor nanoparticles with surface passivation and surface plasmon. Electron. Mater. Lett. 7, 185 (2011).

45. Kim, I. S. et al. Influence of stoichiometry on the optical and electrical properties of chemical vapor deposition derived $\mathrm{MoS}_{2}$. ACS Nano 8, 10551-10558 (2014)

46. Yang, K. et al. The influence of surface chemistry and size of nanoscale graphene oxide on photothermal therapy of cancer using ultra-low laser power. Biomaterials 33, 2206-2214 (2012).

47. Feng, L. et al. Polyethylene glycol and polyethylenimine dual-functionalized nano-graphene oxide for photothermally enhanced gene delivery. Small 9, 1989-1997 (2013).

48. Venkatesan, R., Periasamy, N. \& Srivastava, T. Singlet molecular oxygen quantum yield measurements of some porphyrins and metalloporphyrins. Proc. Indian Acad. Sci. Chem. Sci. 104, 713-722 (1992).

49. Xiao, L., Gu, L., Howell, S. B. \& Sailor, M. J. Porous silicon nanoparticle photosensitizers for singlet oxygen and their phototoxicity against cancer cells. ACS Nano 5, 3651-3659 (2011).

50. Feng, G., Wu, W., Xu, S. \& Liu, B. Far red/near-infrared AIE dots for imageguided photodynamic cancer cell ablation. ACS Appl. Mat. Interfaces 8, 21193-21200 (2016).

51. Matheson, I. B. C. et al. The quenching of singlet oxygen by amino acids and proteins. Photochem. Photobiol. 21, 165-171 (1975).

52. Cheng, L., Wang, C., Feng, L., Yang, K. \& Liu, Z. Functional nanomaterials for phototherapies of cancer. Chem. Rev. 114, 10869-10939 (2014).

53. Peng, F. et al. Silicon nanomaterials platform for bioimaging, biosensing, and cancer therapy. Acc. Chem. Res. 47, 612-623 (2014).

54. Ge, J. et al. A graphene quantum dot photodynamic therapy agent with high singlet oxygen generation. Nat. Commun. 5, 4596 (2014).

55. Lucky, S. S., Soo, K. C. \& Zhang, Y. Nanoparticles in photodynamic therapy. Chem. Rev. 115, 1990-2042 (2015).

56. Seidl, C. et al. Tin Tungstate nanoparticles: a photosensitizer for photodynamic tumor therapy. ACS Nano 10, 3149-3157 (2016).

57. Zhou, Z., Song, J., Nie, L. \& Chen, X. Reactive oxygen species generating systems meeting challenges of photodynamic cancer therapy. Chem. Soc. Rev. 45, 6597-6626 (2016).

58. Bakalova, R., Ohba, H., Zhelev, Z., Ishikawa, M. \& Baba, Y. Quantum dots as photosensitizers? Nat. Biotechnol. 22, 1360 (2004).

59. Samia, A. C. S., Dayal, S. \& Burda, C. Quantum dot-based energy transfer: perspectives and potential for applications in photodynamic therapy. Photochem. Photobiol. 82, 617-625 (2006).

60. Chen, Y. L. et al. Switching luminescent properties in osmium-based $\beta$-diketonate complexes. Chemphyschem 6, 2012-2017 (2005)

61. $\mathrm{Xu}, \mathrm{S}$. et al. Tuning the singlet-triplet energy gap: a unique approach to efficient photosensitizers with aggregation-induced emission (AIE) characteristics. Chem. Sci. 6, 5824-5830 (2015).

62. Zhen, X. et al. Intraparticle energy level alignment of semiconducting polymer nanoparticles to amplify chemiluminescence for ultrasensitive in vivo imaging of reactive oxygen species. ACS Nano 10, 6400-6409 (2016).

63. Chia, S. L., Tay, C. Y., Setyawati, M. I. \& Leong, D. T. Biomimicry 3D gastrointestinal spheroid platform for the assessment of toxicity and inflammatory effects of zinc oxide nanoparticles. Small 11, 702-712 (2015)

64. Li, B. L. et al. Directing assembly and disassembly of $2 \mathrm{D} \mathrm{MoS}_{2}$ nanosheets with DNA for drug delivery. ACS Appl. Mater. Interfaces 9, 15286-15296 (2017).

65. Tay, D. M. Y., Li, B. L., Tan, E. S. L., Loh, K. P. \& Leong, D. T. Precise singlestep electrophoretic multi-sized fractionation of liquid-exfoliated nanosheets. Adv. Funct. Mater. 28, 1801622 (2018).

66. Li, B. L. et al. Emerging OD transition-metal dichalcogenides for sensors, biomedicine, and clean energy. Small 13, 1700527 (2017).
67. Li, B. L. et al. Low-dimensional transition metal dichalcogenide nanostructures based sensors. Adv. Funct. Mater. 26, 7034-7056 (2016).

68. Kresse, G. \& Furthmüller, J. Efficient iterative schemes for ab initio totalenergy calculations using a plane-wave basis set. Phys. Rev. B. Condens. Matter 54, 11169-11186 (1996).

69. Perdew, J. P., Burke, K. \& Ernzerhof, M. Generalized gradient approximation made Simple. Phys. Rev. Lett. 77, 3865 (1996).

70. Alexandre Tkatchenko and Matthias Scheffler.. Accurate molecular van der waals interactions from ground-state electron density and free-atom reference data. Phys. Rev. Lett. 102, 073005 (2009).

71. Gong, Wenbin, Zhang, Wei, Wang, Chengbin, Yao, Yagang \& Lu, Weibang Influence of self-consistent screening and polarizability contractions on interlayer sliding behavior of hexagonal boron nitride. Phys. Rev. B 96, 174101 (2017).

\section{Acknowledgements}

We acknowledge the funding provided by the National Research Foundation, Prime Minister's Office, Singapore, under Competitive Research Program (Award No. NRFCRP13-2014-03).

\section{Author contributions}

X.D. and D.T.L. conceived the project, the hypotheses and the experiments. X.D., F.P., W.B.G., Z.J. performed the experiments. X.D., C.T.L. and D.T.L. analyzed the results. X.D., F.P., G.S., K.P.L., C.T.L., and D.T.L. discussed the results. X.D., C.T. L. and D.T.L. wrote the manuscript.

\section{Additional information}

Supplementary Information accompanies this paper at https://doi.org/10.1038/s41467018-07835-1.

Competing interests: The authors declare no competing interests.

Reprints and permission information is available online at http://npg.nature.com/ reprintsandpermissions/

Journal Peer Review Information: Nature Communications thanks the anonymous reviewers for their contributions to the peer review of this work. [Peer reviewer reports are available].

Publisher's note: Springer Nature remains neutral with regard to jurisdictional claims in published maps and institutional affiliations.

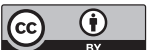

Open Access This article is licensed under a Creative Commons Attribution 4.0 International License, which permits use, sharing, adaptation, distribution and reproduction in any medium or format, as long as you give appropriate credit to the original author(s) and the source, provide a link to the Creative Commons license, and indicate if changes were made. The images or other third party material in this article are included in the article's Creative Commons license, unless indicated otherwise in a credit line to the material. If material is not included in the article's Creative Commons license and your intended use is not permitted by statutory regulation or exceeds the permitted use, you will need to obtain permission directly from the copyright holder. To view a copy of this license, visit http://creativecommons.org/ licenses/by/4.0/.

(C) The Author(s) 2019 